\title{
Tracing the energetics and evolution of dust with Spitzer: a chapter in the history of the Eagle Nebula
}

\author{
N. Flagey ${ }^{1,2}$, F. Boulanger ${ }^{2}$, A. Noriega-Crespo ${ }^{1}$, R. Paladini ${ }^{1}$, T. Montmerle $^{3,4}$, \\ S. J. Carey ${ }^{1}$, M. Gagné ${ }^{5}$, and S. Shenoy ${ }^{1,6}$
}

\author{
1 Spitzer Science Center, California Institute of Technology, 1200 East California Boulevard, MC 220-6, Pasadena, CA 91125, USA \\ e-mail: nflagey@jpl.nasa.gov \\ 2 Institut d'Astrophysique Spatiale, Université Paris Sud, Bât. 121, 91405 Orsay Cedex, France \\ 3 Institut de Planétologie et d'Astrophysique de Grenoble, BP53, 38041 Grenoble Cedex 9, France \\ 4 Institut d'Astrophysique de Paris, 98bis Bd Arago, 75014 Paris, France \\ 5 Department of Geology and Astronomy, West Chester University, West Chester, PA 19383, USA \\ ${ }^{6}$ Space Science Division, Mail Stop 245-6, NASA Ames Research Center, Moffett Field, CA 94035, USA
}

Received 5 January 2011 / Accepted 10 April 2011

\begin{abstract}
Context. The Spitzer GLIMPSE and MIPSGAL surveys have revealed a wealth of details about the Galactic plane in the infrared (IR) with orders of magnitude higher sensitivity, higher resolution, and wider coverage than previous IR observations. The structure of the interstellar medium (ISM) is tightly connected to the countless star-forming regions. We use these surveys to study the energetics and dust properties of the Eagle Nebula (M 16), one of the best known star-forming regions.

Aims. We present MIPSGAL observations of M 16 at 24 and $70 \mu \mathrm{m}$ and combine them with previous IR data. The mid-IR image shows a shell inside the well-known molecular borders of the nebula, as in the ISO and MSX observations from 15 to $21 \mu \mathrm{m}$. The morphologies at 24 and $70 \mu \mathrm{m}$ are quite different, and its color ratio is unusually warm. The far-IR image resembles the one at $8 \mu \mathrm{m}$ that enhances the structure of the molecular cloud and the "pillars of creation". We use this set of IR data to analyze the dust energetics and properties within this template for Galactic star-forming regions.

Methods. We measure IR spectral energy distributions (SEDs) across the entire nebula, both within the inner shell and the photodissociation regions (PDRs). We use the DUSTEM model to fit these SEDs and constrain the dust temperature, the dust-size distribution, and the radiation field intensity relative to that provided by the star cluster NGC $6611\left(\chi / \chi_{0}\right)$.

Results. Within the PDRs, the inferred dust temperature $(\sim 35 \mathrm{~K})$, the dust-size distribution, and the radiation field intensity $\left(\chi / \chi_{0}<1\right)$ are consistent with expectations. Within the inner shell, the dust is hotter $(\sim 70 \mathrm{~K})$. Moreover, the radiation field required to fit the SED is larger than that provided by NGC $6611\left(\chi / \chi_{0}>1\right)$. We quantify two solutions to this problem: (1) The size distribution of the dust in the shell is not that of interstellar dust. There is a significant enhancement of the carbon dust-mass in stochastically heated very small grains. (2) The dust emission arises from a hot $\left(\sim 10^{6} \mathrm{~K}\right)$ plasma where both UV and collisions with electrons contribute to the heating. Within this hypothesis, the shell SED may be fit for a plasma pressure $p / k \sim 5 \times 10^{7} \mathrm{~K} \mathrm{~cm}^{-3}$.

Conclusions. We suggest two interpretations for the M 16 inner shell: (1) The shell matter is supplied by photo-evaporative flows arising from dense gas exposed to ionized radiation. The flows renew the shell matter as it is pushed out by the pressure from stellar winds. Within this scenario, we conclude that massive-star forming regions such as M 16 have a major impact on the carbon dustsize distribution. The grinding of the carbon dust could result from shattering in grain-grain collisions within shocks driven by the dynamical interaction between the stellar winds and the shell. (2) We also consider a more speculative scenario where the shell is a supernova remnant. In this case, we would be witnessing a specific time in the evolution of the remnant where the plasma pressure and temperature would enable the remnant to cool through dust emission.
\end{abstract}

Key words. HII regions - dust, extinction - photon-dominated region (PDR) - ISM: individual objects: Eagle Nebula - ISM: bubbles

\section{Introduction}

The Eagle Nebula (M 16) is a nearby $(d=2.0 \pm 0.1 \mathrm{kpc}$, Hillenbrand et al. 1993) massive-star forming region that has become an icon since the publication of spectacular Hubble Space Telescope (HST) images of its ionized gas emission (Hester et al. 1996). As one of the nearest star-forming regions and one of the most well-observed across the electromagnetic spectrum, the Eagle Nebula is a reference source. The nebula cavity is carved into the molecular cloud by a cluster of 22 ionizing stars earlier than B3 (Dufton et al. 2006b) with an estimated age of $1-3 \times 10^{6}$ yr (Hillenbrand et al. 1993; Dufton et al. 2006b; Martayan et al. 2008).
The mid-IR images of M 16 from either the Infrared Space Observatory CAMera (ISOCAM Cesarsky et al. 1996a) at 8 and $15 \mu \mathrm{m}$ (Pilbratt et al. 1998; Omont et al. 2003) or based on the combined Spitzer observations using IRAC $8 \mu \mathrm{m}$ (Fazio et al. 2004) and MIPS $24 \mu \mathrm{m}$ (Rieke et al. 2004), show a shell-like emission at 15 and $24 \mu \mathrm{m}$ that fills the nebula cavity (Flagey et al. 2009a), as delineated by the shorter IR wavelengths and the extent of the $\mathrm{H}_{\alpha}$ emission. The shell stands out in the ISO $15 \mu \mathrm{m}$ and MIPS $24 \mu \mathrm{m}$ images, while the Nebula pillars and the outer rim of the nebula are the strongest emission features at $8 \mu \mathrm{m}$. On the basis of spectroscopic evidence (Urquhart et al. 2003), it is known that the mid-IR shell emission is 
produced by dust, there being only a minor contribution from ionized gas lines to the broadband emission.

M 16 is not alone in this respect. There are other large, partially symmetrical and rich HII regions (in terms of their OB stellar content) that display a similar mid-IR color stratification: the Rosette Nebula (Kraemer et al. 2003), the Trifid Nebula (Lefloch et al. 1999; Rho et al. 2006), and M 17 (Povich et al. 2007). Furthermore, the multi-wavelength observations of the HII regions in the Galactic Plane, using the Spitzer GLIMPSE and MIPSGAL Legacy surveys (Churchwell et al. 2009; Carey et al. 2009) display overall a wide variety of complex morphologies, and contain many "bubble"-like objects with a similar color stratification as M 16 (Watson et al. 2008, 2009), although they are smaller and driven by one or a few OB stars.

What do these Spitzer images of massive-star forming regions teach us about dust and the interaction of the stars with their environment? The IRAC and the MIPS $24 \mu \mathrm{m}$ camera image the emission from PAHs and very small grains (VSGs). A first key to the interpretation of Spitzer images is the change in abundance and excitation of these small dust particles from molecular to ionized gas. Observations of nearby molecular clouds illuminated by $\mathrm{O}$ stars, where observations separate the $\mathrm{H}$ II photo-ionized gas layer from the neutral photo-dissociation region (PDR) show that the PAH bands, which are a characteristic of PDR mid-IR emission spectra, are strikingly absent from that of the H II layer (e.g., the Orion Bar and the M 17SW interface, Giard et al. 1994; Cesarsky et al. 1996b; Povich et al. 2007). PAHs are quickly destroyed when matters flows across the ionization front. Several destruction mechanisms have been proposed such as the chemisputtering by protons and photothermo dissociation and/or Coulomb explosion associated with the absorption of high energy photons. Much less is known about the evolution of VSGs. The mid-IR shells may reflect dust processing by hard photons and shocks that impact the fraction of the dust mass in VSGs, but this possibility has yet to be constrained by modeling the dust emission.

The evolutionary stage of the massive-star forming regions is a second key to the interpretation of the Spitzer images. The mid-IR shells do not fit the classical view of the evolution of HII regions where the matter is swept away by the simultaneous effect of the ionization, stellar winds, and radiation pressure from their central OB stars (e.g. Tenorio-Tagle et al. 1982; Beltrametti et al. 1982; Rozyczka 1985). In this scenario, the HII regions are "hollow". One interesting possibility is that gas photo-evaporating from dense condensations exposed to ionized radiation, creates a gas mass input within the cavity sufficient to balance the outward flow of matter. Do the shells reflect this mass input? To show that this is a plausible interpretation, one must quantify the mass input, as well as the dust properties and excitation conditions, required to match the shell brightness and its distinct mid-IR colors.

Most studies on the mid-IR properties of these HII regions and smaller bubbles have to date been phenomenological and have considered the spatial distribution of the different emission components and not physics. A small bubble where a more quantitative analysis has been carried out is G28.82-0.23 ( $a \mathrm{ka}$ N49, Everett \& Churchwell 2010) which is nearly spherically symmetric, is excited by a single O5V star, and has a thick $8 \mu \mathrm{m}$ shell surrounding at $24 \mu \mathrm{m}$ a diffuse bubble (see e.g. Watson et al. 2008, Fig. 7). Everett \& Churchwell (2010) proposed a model where the mid-IR emission of G28.82-0.23 arises from dust entrained by the stellar wind. This interpretation involves a hot $\left(>10^{6} \mathrm{~K}\right)$, high pressure plama $\left(p / k \sim 10^{9} \mathrm{~K} \mathrm{~cm}^{-3}\right)$ where the dust lifetime is shorter than the expansion timescale. This implies that the dust is constantly replenished by the photoevaporation of high density $\left(10^{5} \mathrm{~cm}^{-3}\right)$ dusty gas cloudlets that have been overrun by the expanding nebula. Collisional excitation by hot electrons contribute significantly to the heating of dust. Infrared dust emission is the dominant cooling channel of the dusty wind, which reduces the energy available for winddriven expansion. This specific model does not appear to offer a general framework to interpret observations of larger HII regions, where one observes a similar 8 and $24 \mu \mathrm{m}$ color stratification.

The motivation of this paper is to study the nature of mid-IR shells in massive-star forming regions using the Eagle Nebula as a template source. The detailed data available on this nearby nebula allow us to perform a quantitative modeling of the dust heating by UV radiation and, possibly, by collisions in a hot plasma. We quantify the dust emission in terms of dust physics, before discussing possible interpretations within an evolutionary scenario of the Eagle Nebula as a massive-star forming region. In Sect. 2, we present the Spitzer imaging observations of the Eagle Nebula from the MIPSGAL Galactic plane survey. Section 3 describes the morphology of M 16 based on IR photometric and spectroscopic observations. We measure the spectral energy distributions (SEDs) across the entire nebula combining data from the ISO, MSX, and Spitzer space missions. In Sects. 4 and 5, we present modeling of the dust properties. We first model the dust SEDs with UV heating only, and this constrains the radiation field intensity and dust-size distribution. We then consider the possibility that the shell emission arises from a hot plasma where dust would be heated by collisions with electrons. The reader not interested in the details of the modeling can skip Sects. 4 and 5. In Sect. 6, we propose two scenarios of the present evolutionary state of the Eagle Nebula, which could account for the mid-IR shell and fit within present observational constraints. The paper results are summarized in Sect. 7.

\section{Observations}

The Eagle Nebula was observed by the Spitzer Space Telescope as part of the GLIMPSE (program \#00146, Benjamin et al. 2003) and MIPSGAL (program \#205976, Carey et al. 2009) inner Galaxy surveys. The GLIMPSE survey used the InfraRed Array Camera (IRAC, Fazio et al. 2004), while MIPSGAL was compiled using the Multiband Imaging Photometer for Spitzer (MIPS, Rieke et al. 2004). In both cases, we used their enhanced products (Squires et al. 2005). The MIPSGAL $24 \mu \mathrm{m}$ data were complemented with archival observations (Spitzer program \#20726) and reprocessed using the standard Spitzer Post-Basic Calibrated Data tools ${ }^{1}$. A three-color image combining IRAC and MIPS data is shown in Fig. 1.

Most of the data processing performed on the MIPSGAL $24 \mu \mathrm{m}$ observations is described in Mizuno et al. (2008) and Carey et al. (2009). At $70 \mu \mathrm{m}$, Spitzer detectors are Ge:Ga photoconductors. When observing bright, structured emission, such as that in the Eagle Nebula, these detectors display significant variations in responsivity, which manifest themselves as visible stripes in the final images and result in photometric errors of several tens of percent. This effect has required an offline reprocessing of the data, with tools specifically designed to, at the same time, reconstruct the history-dependent responsivity variations of the detectors and mitigate the associated stripes. The photometric uncertainty of the extended emission is reduced from

1 http://ssc.spitzer.caltech.edu/postbcd/ 
N. Flagey et al.: Tracing the energetics and evolution of dust with Spitzer: a chapter in the history of the Eagle Nebula

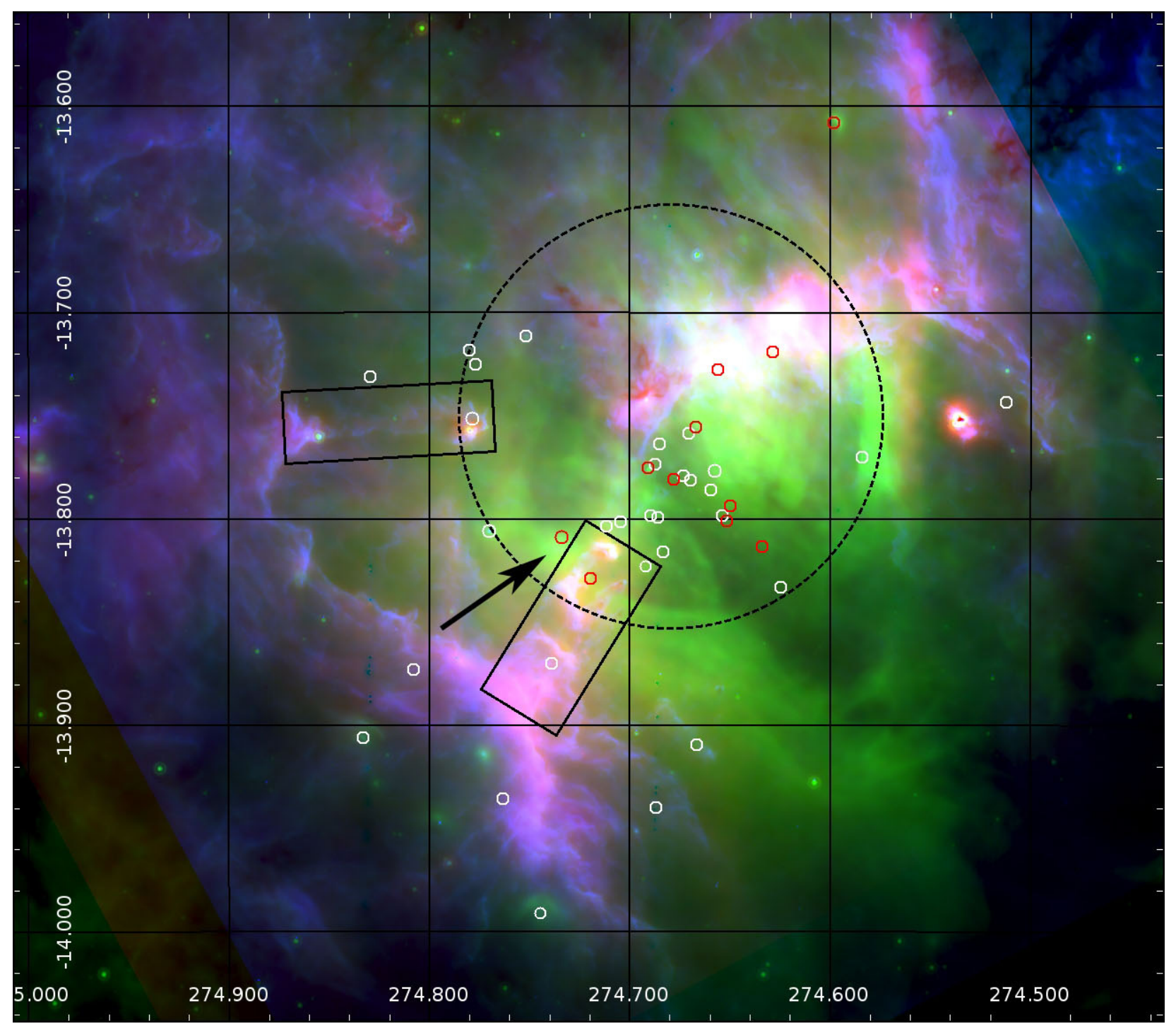

(a)

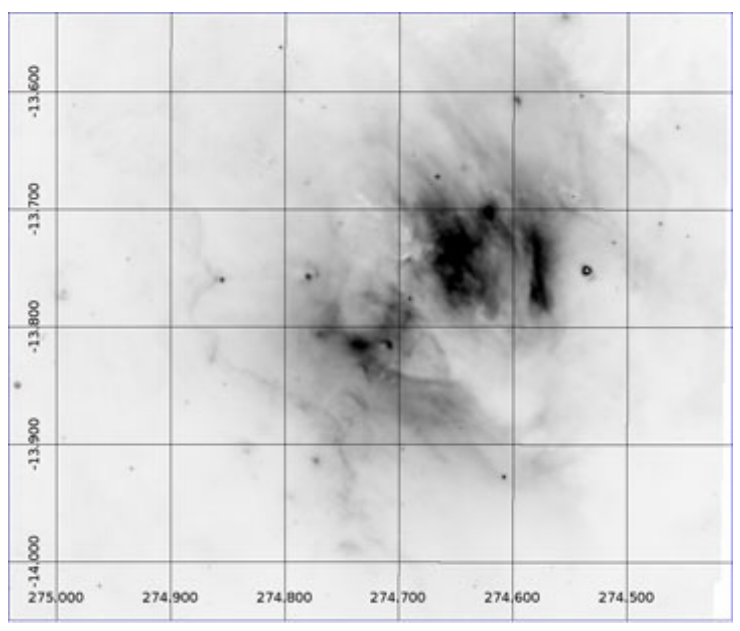

(b)

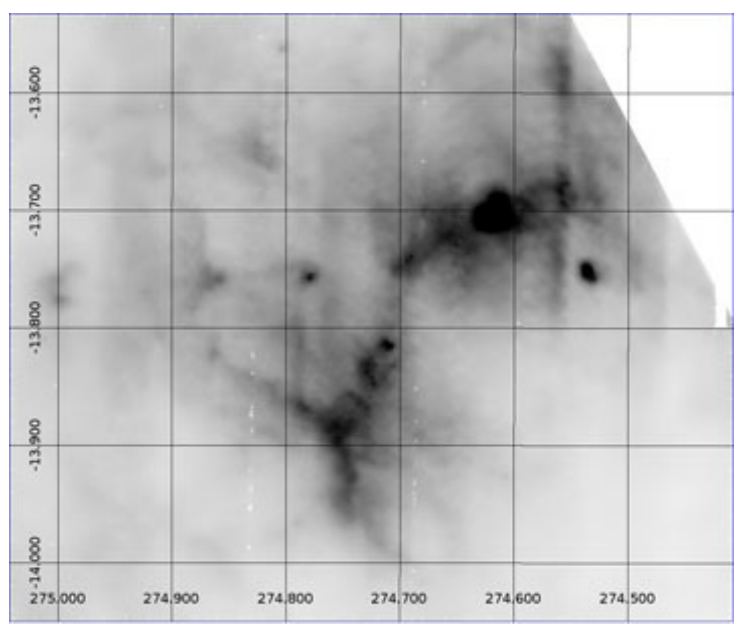

(c)

Fig. 1. a) Composite Spitzer color image combining the IRAC $8.0 \mu \mathrm{m}$ (blue) bands with MIPS 24 (green) and $70 \mu \mathrm{m}$ (red). The FOV is $\sim 30^{\prime}$, N is up and $\mathrm{E}$ is left. The two black boxes outline the "pillars of creation", which extend from the bottom to the center, pointing slightly to the W, and the "spire", originating in the E and almost pointing straight toward the W. The black circle outlines the MIPS $24 \mu \mathrm{m}$ shell. The black arrow indicates the position of Pilbratt's blob. The position and spectral type of the most massive stars of NGC 6611 are overplotted: O stars are in red, B stars are in white. The MIPS 24 and $70 \mu$ m images are also shown separately in panels b) and c). 


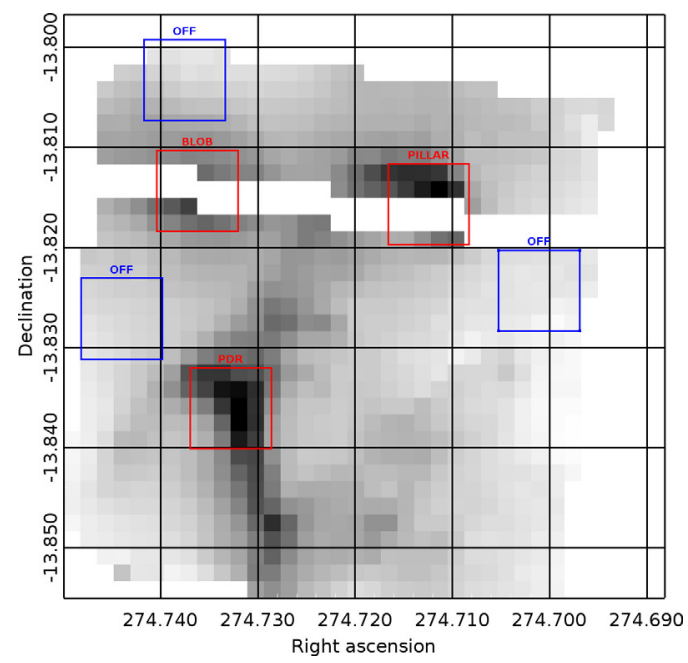

(a)

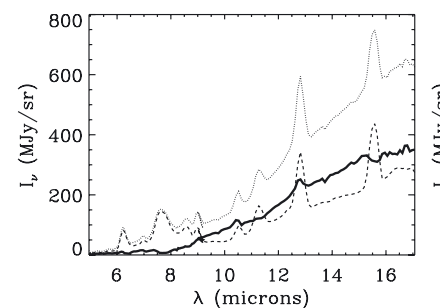

(b)

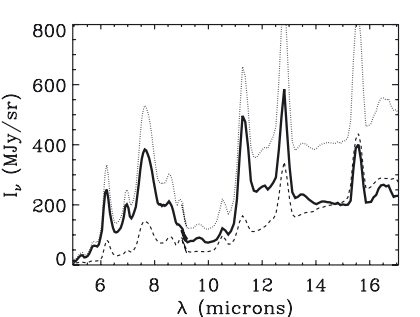

(c)

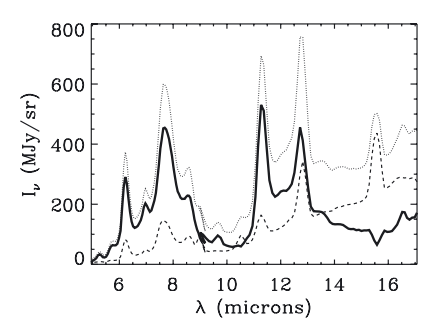

(d)

Fig. 2. ISOCAM/CVF mean spectra observed a) on Pilbratt's blob, b) at the tip of the main "pillar of creation", and c) on a PDR within the "pillars of creations". Dotted lines are ON spectra, dashed lines are OFF spectra, and thick solid lines are ON-OFF spectra. OFF and ON positions are shown for the ISOCAM/CVF $3^{\prime}$ by $3^{\prime}$ field of view, here at the wavelength of $12 \mu \mathrm{m}$. North is up, East to the left.

about $50 \%$ for the brightest features to about $15 \%$ for the enhanced MIPS $70 \mu \mathrm{m}$ data. The specific pipeline developed for the MIPSGAL $70 \mu \mathrm{m}$ observations will be described in Paladini et al. (in prep.).

We combine the Spitzer observations of M 16 with a previous IR survey from MSX and observations from ISO, both photometric and spectroscopic. The ISOCAM/CVF spectra have already been presented by Urquhart et al. (2003). A slice of the ISOCAM/CVF spectroscopic cube is shown in Fig. 2a.

\section{Observational results}

We use the many IR observations available to create a portrait of the nebula from NIR to FIR wavelengths. We then perform aperture measurements on both the broad band images and spectroscopic observations to derive characteristic SEDs and spectra of the Eagle Nebula. We focus our comments on the two main features of the nebula: the PDRs and the inner shell.

\subsection{Images}

The three-color image of Fig. 1 clearly highlights differences between intermediate wavelengths on the one hand (MIPS24 in green) and the shorter and longer wavelengths on the other (IRAC8 in blue and MIPS70 in red). The whole molecular cloud appears in purple, while the inner shell is green. These differences include the following:

- at wavelengths shorter than $\sim 10 \mu \mathrm{m}$, IRAC, MSX, and ISO observations show the molecular cloud surface heated by the cluster UV radiation. The "pillars of creation", the "spire" (see Fig. 1 to identify these structures), and emission of lower contrast extends towards the cluster from the $\mathrm{N}$ and the E. To the NW and the SE, the rim of an outer shell can be identified. It corresponds to the edge of the Eagle Nebula as seen in $\mathrm{H} \alpha$;

- at intermediate wavelengths, between $\sim 12$ and $24 \mu \mathrm{m}$, MSX, ISO, and MIPS observations exhibit a significantly distinct morphology, with a shell filling the inside cavity in-between the pillars and the edges of the molecular cloud seen at shorter wavelengths. The shell extends over $\sim 12^{\prime}$ in the NWSE direction towards the pillars and further out to the SW where there is no emission at either shorter or longer wavelengths. There are some bright features within the shell, some of which have already been identified (e.g. Pilbratt's blob, to the E of the main "pillar of creation", Pilbratt et al. 1998). The lack of far-infrared observations prevented previous authors from concluding anything specific about the nature of this shell;

- at longer wavelengths, the MIPS $70 \mu \mathrm{m}$ observations are very similar to those at shorter wavelengths and mainly show the molecular cloud surface. The diffuse emission within the inside cavity is visible but not as bright as at intermediate wavelengths. The lower angular resolution of these observations does not allow us to make more detailed comments at this point.

The IR morphology of the Eagle Nebula is common among other star-forming regions. Churchwell et al. (2006) listed many of these "bubbles" across the entire GLIMPSE Galactic plane survey with IRAC. Combining GLIMPSE and MIPSGAL $24 \mu \mathrm{m}$ surveys reveals that an inner shell exists in most of these regions $^{2}$.

\subsection{SED measurements}

We perform ON-OFF aperture measurements to get both spectroscopic and photometric SEDs. As shown in Fig. 2a, there is a band of unavailable pixels in the ISOCAM/CVF observations. This band goes exactly through interesting and features of sharp contrast such as the tip of the main pillar and Pilbratt's blob. Rather than linearly interpolate the missing pixels as Urquhart et al. (2003) did previously for ISOCAM/CVF data, we use these data as they are. We present and interpret spectroscopic and photometric measurements separately.

\subsubsection{Spectroscopic measurements}

We compute average spectra at multiple positions within the "pillars of creation" area covered by the ISOCAM/CVF data. We use square boxes of $4 \times 4$ pixels $(24 \times 24$ " on

\footnotetext{
2 http://www. spitzer. caltech.edu/Media/releases/ ssc2008-11/ssc2008-11a.shtml
} 
ISOCAM/CVF 6" pixel field of view) to estimate the mean brightness of several features. We use this method for both "ON" and "OFF" positions. We combine three different OFF positions to build a unique OFF spectrum. The resulting ON-OFF spectra are shown in Fig. 2 for two positions within the main "pillar of creation" and one on Pilbratt's blob. These three positions, marked in Fig. 2a, correspond, respectively, to spectra D, B, and A of Fig. 2 from Urquhart et al. (2003). One of our OFF positions is close to their spectrum $\mathrm{C}$. As a consequence, our results are similar to theirs:

- the spectra of the "pillars of creation" (see Figs. 2c and d) exhibit the characteristics of PDR spectra with strong PAH features and gas lines. They also present the Si absorption feature around $10 \mu \mathrm{m}$. There are some variations between the two positions, mainly in terms of PAH features and gas line strength, which traces variations in the excitation conditions between these two positions within the column of gas and dust;

- the spectrum of Pilbratt's blob (see Fig. 2b) exhibits a strong continuum with very weak gas lines and PAHs bands. We thus assume, as a first approximation, that the MIPS $24 \mu \mathrm{m}$ shell is dust-continuum dominated;

- the OFF position has a spectrum with a weaker continuum than the blob but stronger than the pillars. It has also much weaker lines and features than the gaseous, dusty columns.

\subsubsection{Photometric measurements}

We combine IR observations of the Eagle Nebula from three different observatories: MSX, ISO, and Spitzer. Therefore, we first lower the spatial resolution of each observations to that of the MSX data $\left(20^{\prime \prime}\right)$. Then, as we did with the spectroscopic measurements, we identify several interesting and high-contrast features within the nebula. We name them as follows. The "PDR" group of features contains the tip of the main "pillar of creation" ("pillar", also known as Col. I, with an embedded source at its tip, see Fig. 3a), the tip of the "spire" ("spire", also known as Col. IV, with an embedded source at its tip, see Fig. 4a) and a PDR within the main "pillar of creation" ("shoulder", see Fig. 5a). The "shell" group of features contains Pilbratt's blob ("blob", see Fig. 6a), the contrasted border of the main shell ("shell border", see Fig. 7a), a diffuse shell that extends towards the opposite direction ("reverse shell", see Fig. 8a), a bright filament on the NW side of the nebula ("filament", see Fig. 9a) and some more diffuse emission on the SW side of the nebula ("diffuse", see Fig. 10a). For each structure, the main difficulty in the measurement is to properly estimate the background emission behind each of them. This is particularly true for the MIPS $70 \mu$ m images.

We illustrate our method for the example of Pilbratt's blob but it is mainly valid for the whole set of structures. We first select a rectangular area that encompasses the blob, as shown in Fig. 6a. We choose the orientation of the selected area so as to avoid selecting other neighboring high-contrast features (e.g. the "pillars of creation"). We then compute the mean profile of the blob and its surroundings by averaging all the pixels along the short axis. The resulting profiles, independently normalized for each band for clarity purposes, for the Pilbratt's blob are shown in Fig. $6 \mathrm{~b}$ for several wavelengths. The profiles for the other features are shown in Figs. 7b through 10b. The MIPS $24 \mu \mathrm{m}$ peak surface brightness for each structure is given in Table 2.

We then measure the mid to far-IR SED of each structure. We adapt the method as a function of the profile shape. For

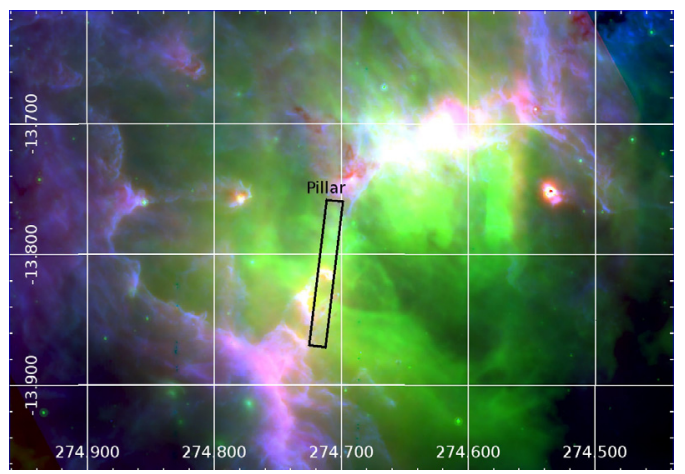

(a)

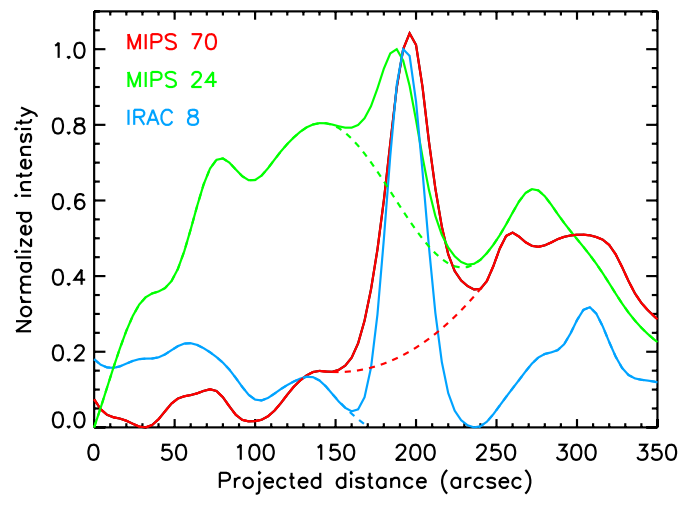

(b)

Fig. 3. a) Three color image as in Fig. 1 with the region along which the profiles are measured for the main "pillar of creation". b) Normalized infrared emission profiles (MIPS70 in red, MIPS24 in green, and IRAC8 in blue, solid lines) and interpolations performed to measure the fluxes of the structure (dashed lines).

the structures that display a peak of emission at every wavelength (e.g. Pilbratt's blob, Spire), we estimate the background by means of a spline interpolation of the profile on both sides of the peak (see Fig. 5b). The flux of the structure is thus given by the integration of the background subtracted profile over the size of the structure. The actual size over which we integrate the flux may slightly vary from one channel to another. The uncertainty in each measurement is given by the range of background values estimated using the spline interpolation. For the other structures, where the profiles exhibit a "jump" (case of the "shell border", see Fig. 7b), we estimate the height of the "jump" at each wavelength by measuring the difference of the surface brightness between the top and bottom of the "jump". The uncertainty in each measurement is given by the standard deviation in the surface brightness at the top and the bottom of the "jump".

While the measurements are usually straightforward for the MIPS $24 \mu \mathrm{m}$ profiles, they are significantly more uncertain for the MIPS $70 \mu \mathrm{m}$ profiles, especially for structures of weaker contrast such as the "filament" or the "diffuse" emission. In those two last cases, we are not sure about the exact spatial extent of the structure at $70 \mu \mathrm{m}$ and the range over which to estimate the background (see Fig. 10b). This generally also applies to the IRAC 8 and $6 \mu \mathrm{m}$ measurements, but to a lesser extent. In particular, for the "filament" structure, the discrepancy in the profile's peak position between MIPS $24 \mu \mathrm{m}$ and MIPS $70 \mu \mathrm{m}$ or IRAC $8 \mu \mathrm{m}$ is significant enough to ensure that we cannor consider them as probing the same physical conditions (see Figs. 9b and 10b). Since there is no other obvious 
A\&A 531, A51 (2011)

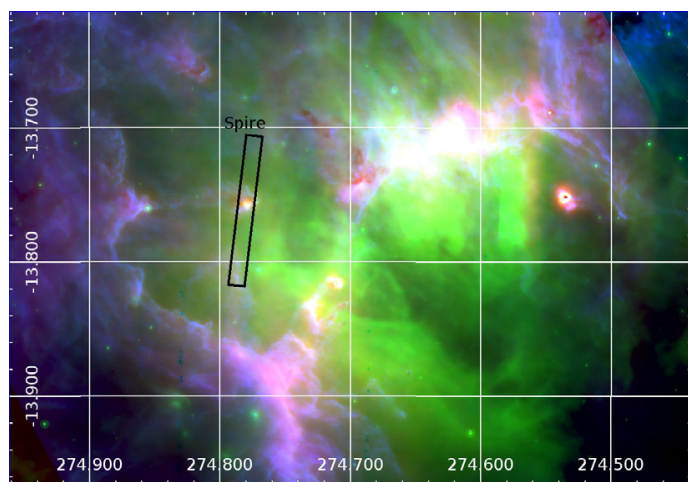

(a)

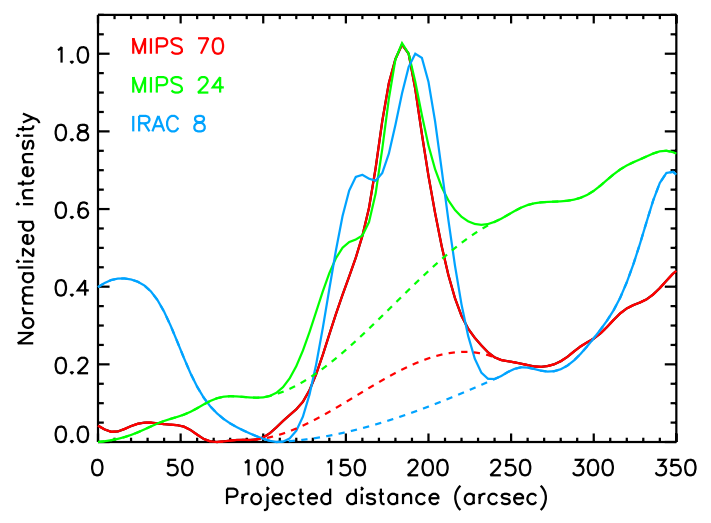

(b)

Fig. 4. Same as Fig. 3 for the position of the "spire".

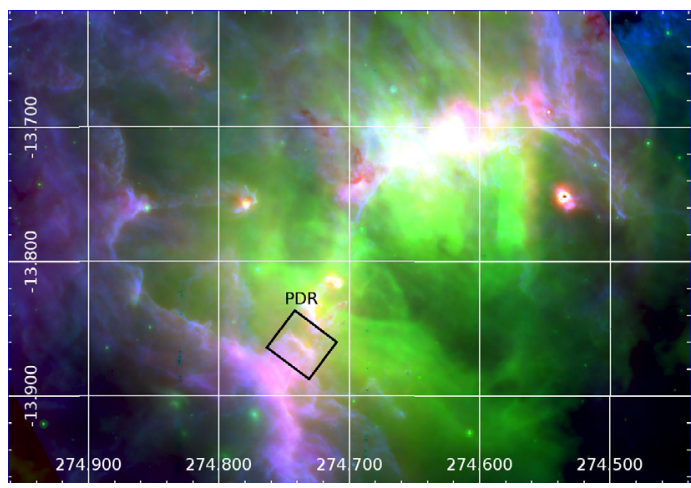

(a)

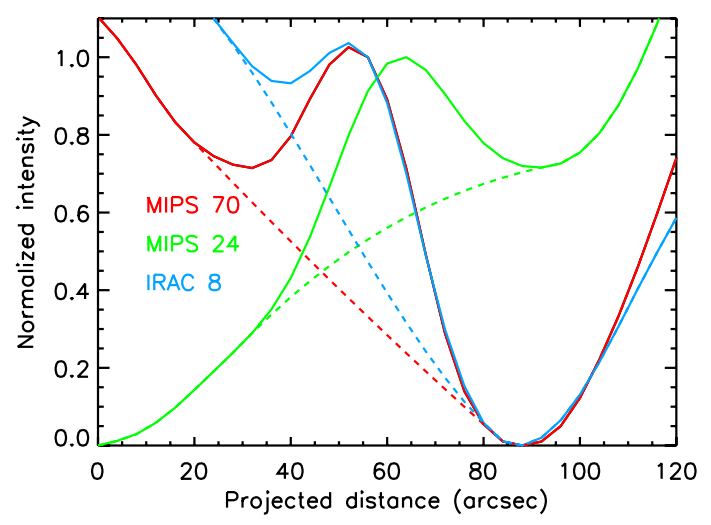

(b)

Fig. 5. Same as Fig. 3 for the position of the "shoulder".

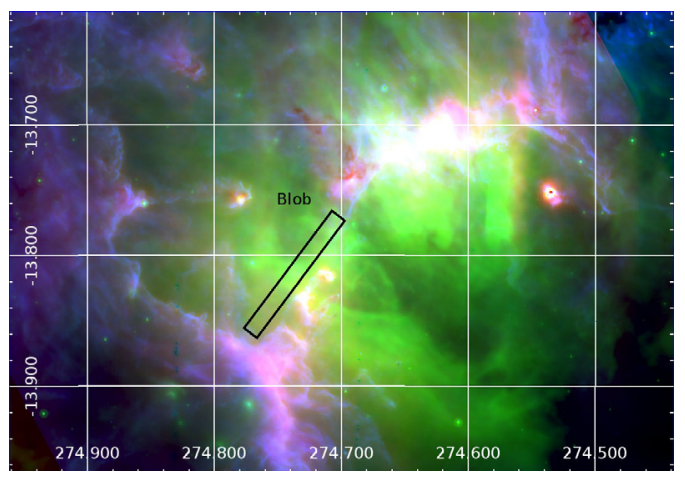

(a)

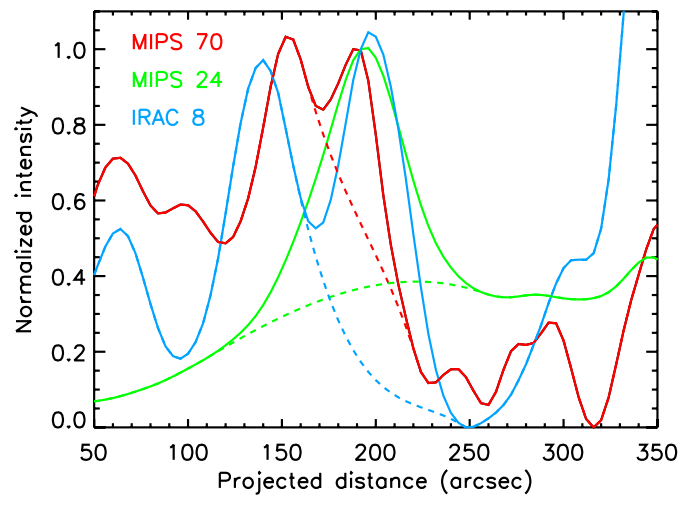

(b)

Fig. 6. Same as Fig. 3 for the position of the "blob".

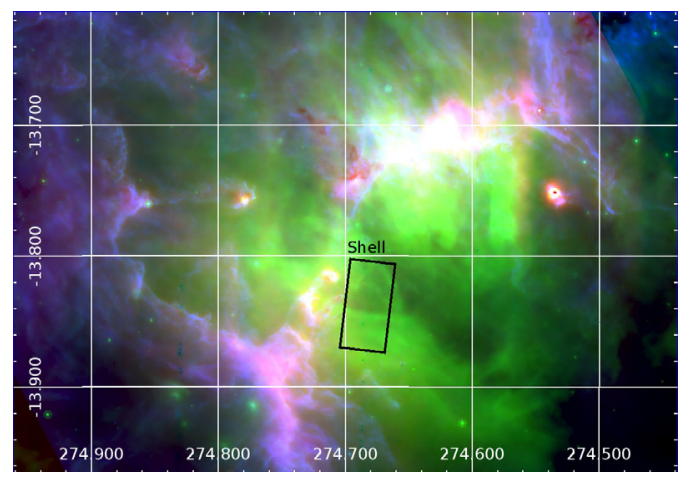

(a)

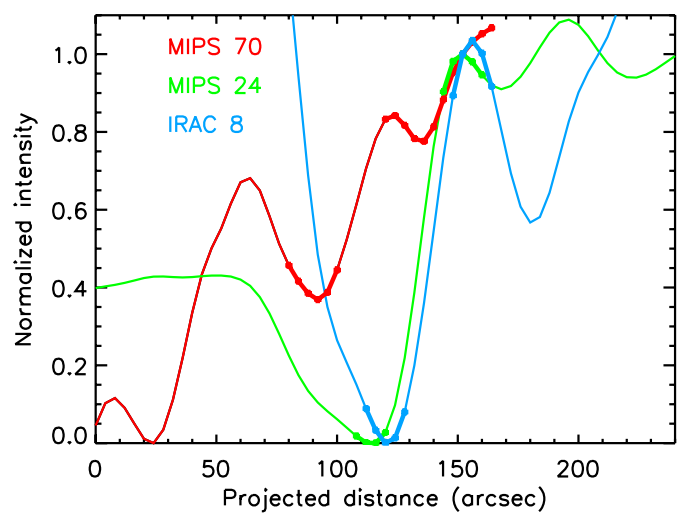

(b)

Fig. 7. Same as Fig. 3 for the position of the "shell border". The darker sections of the profiles show the top and bottom of the "jump" used to measure the fluxes at each wavelength. 
N. Flagey et al.: Tracing the energetics and evolution of dust with Spitzer: a chapter in the history of the Eagle Nebula

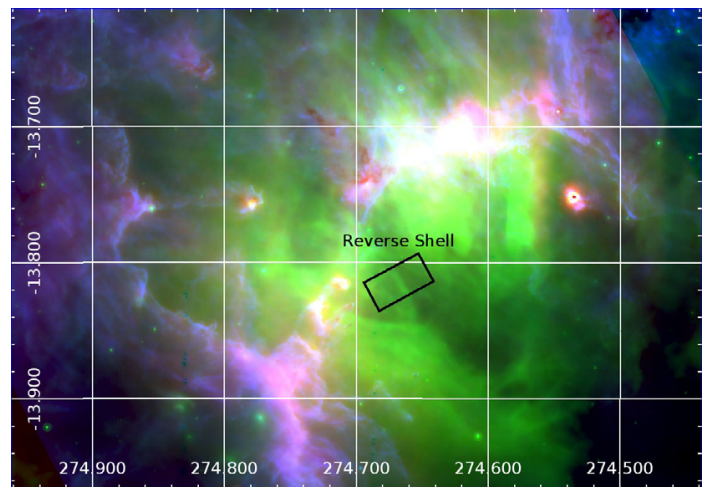

(a)

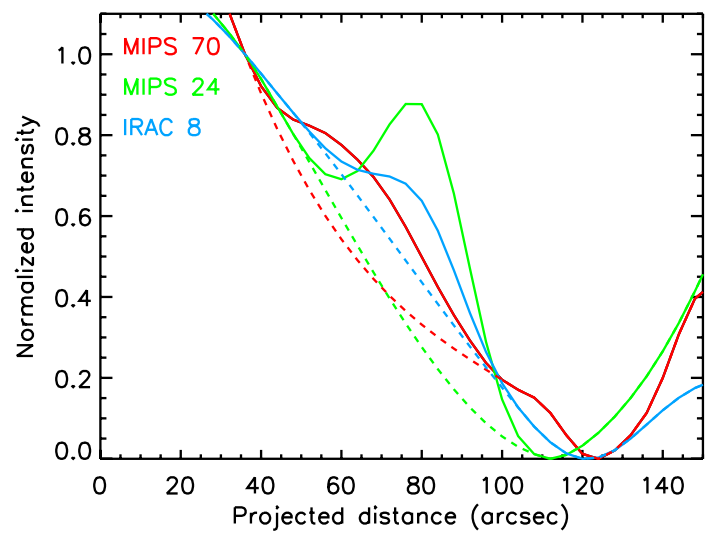

(b)

Fig. 8. Same as Fig. 3 for the position of the "reverse shell".

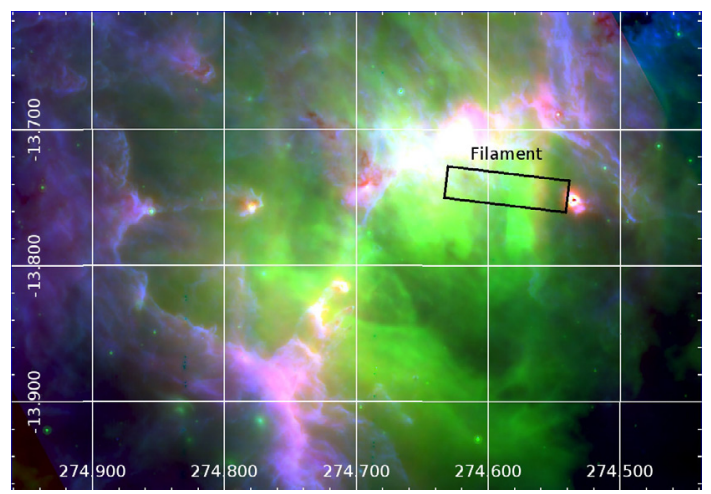

(a)

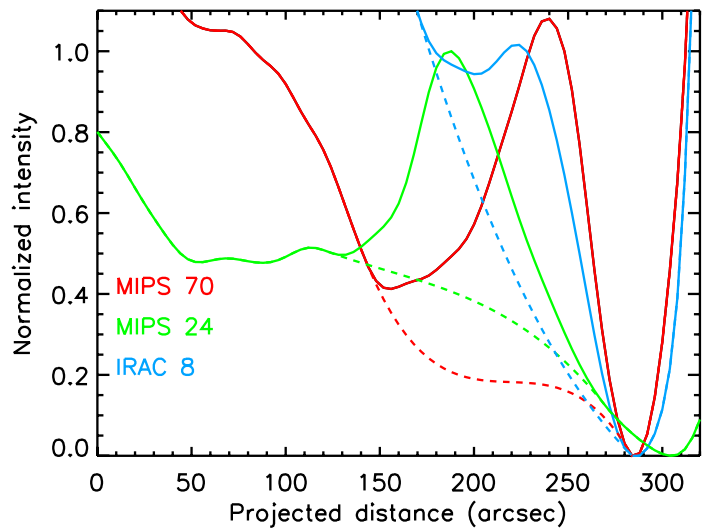

(b)

Fig. 9. Same as Fig. 3 for the position of the "filament".

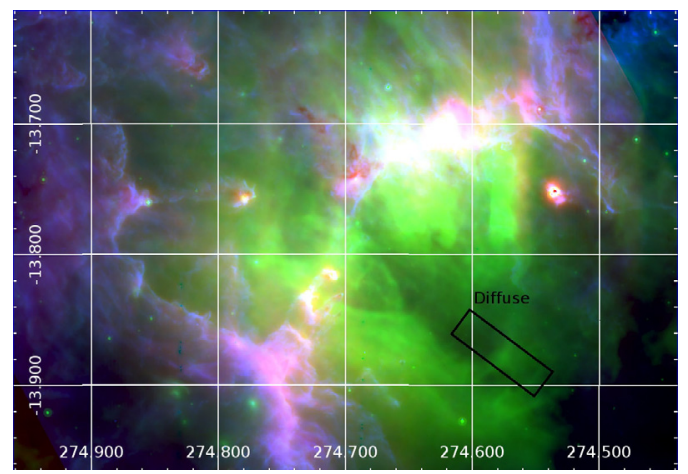

(a)

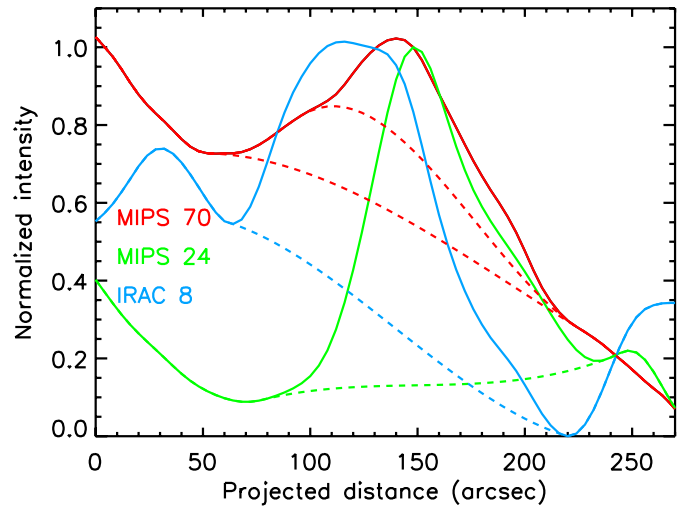

(b)

Fig. 10. Same as Fig. 3 for the position of the "diffuse" emission.

feature at the position of the MIPS $24 \mu \mathrm{m}$ peak, we thus use the MIPS70 $\mu \mathrm{m}$ measurement as an upper limit. In addition, the uncertainty in the MIPS $70 \mu \mathrm{m}$ flux of the "diffuse" emission is significantly higher. The resulting photometric SEDs, normalized to MIPS $24 \mu \mathrm{m}$, are presented in Fig. 11. The differences between the structures within the shell and those within the PDRs are again clear:

- the PDRs of M 16, both at the tip of the "spire" and within the "pillars of creation", are characterized by an almost flat SED from near to mid infrared and a continuous increase from mid to far infrared wavelengths. The SEDs of the position with an embedded source ("pillar" and "spire") do not appear to differe from that of the "shoulder" at near infrared wavelengths. At longer wavelengths, the SED of the "shoulder" increases slightly less than those of the "pillar" and the "spire", which both encompass an embedded source. The ratio of the MIPS24 to MIPS70 mean flux is about 0.1 for the "shoulder" and about 0.3 at the tip of the main "pillar of creation" and the "spire";

- the inside shell, at Pilbratt's blob position and in bright sharp-contrast structures, is characterized by a significantly steeper increase in the intensity from near to mid infrared and a flat or decreasing SED from mid to far infrared. In Pilbratt's blob, the "shell border" and the "reverse shell", the MIPS24 to MIPS 70 ratio is about 4.5, 2.3 and 0.95 respectively;

- the "filament" and the "diffuse" SEDs appear to be inbetween these two sets of SEDs. Both their MIPS24 to MIPS 70 ratio is lower than inside the shell and their near to mid infrared SED is steeper than within PDRs but the uncertainties are significantly larger. As a consequence, in the following sections, we do not discuss these last two positions. 


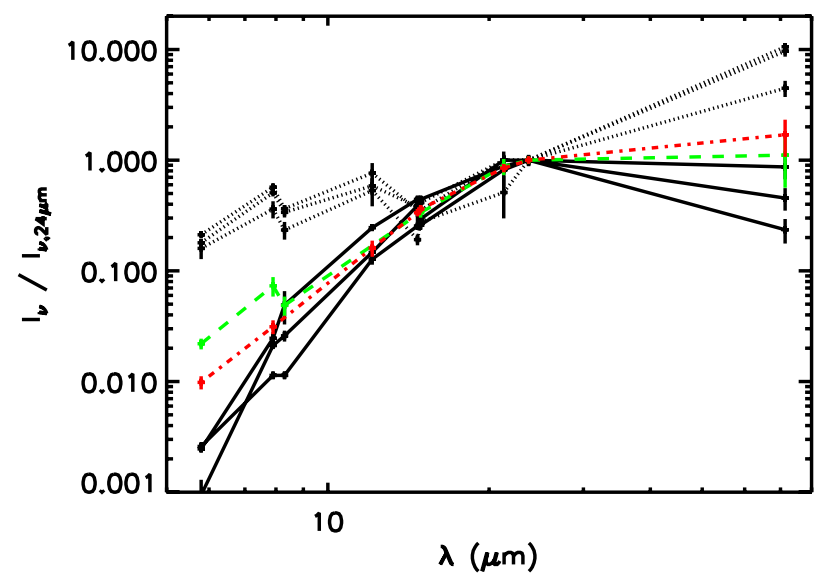

Fig. 11. Comparison of the structure SED. Solid lines: structures within the shell. Dotted lines: structures within the PDRs. Red dash-dotted line: "filament". Green dashed line: "diffuse".

The measurements of the near-IR to far-IR SEDs confirm what spectroscopic observations were suggesting: the dust within the inner shell is significantly different from that within PDRs. The addition of the MIPS $70 \mu \mathrm{m}$ data and its comparison to the MIPS $24 \mu \mathrm{m}$ data provide us with constraints on the position of the dust emission peak in the FIR. We explore in the next section whether the difference arises from external excitation or intrinsic properties.

\section{UV heating of the dust}

We model the dust emission within M 16 using the dust model of Compiègne et al. (2011). In this model, the dust is heated only by the incident flux of UV photons. We first show that the MIPS $24 \mu \mathrm{m}$ to MIPS $70 \mu \mathrm{m}$ ratio may be directly related to the intensity of the radiation field in the shell. We then use the dust model to determine the best-fit set of parameters describing the complete observed SEDs over the entire nebula. In this section, we limit ourselves to the following parameters: the intensity of the incident radiation field and the dust-size distribution, in terms of the abundances of the dust components.

\subsection{Method}

The dust model of Compiègne et al. (2011) is an updated version of the original Désert et al. (1990) model. In their model, Compiègne et al. (2011) use four dust components: (1) polycyclic aromatic hydrocarbons (PAH); (2) stochastically heated very small grains of amorphous carbon (VSG or SamC); (3) large amorphous carbon grains (LamC); and (4) amorphous silicates (aSil). We combine LamC and aSil grains into a unique big grain (BG) component using these grains relative abundances found in the diffuse high galactic latitude (DHGL) medium (Compiègne et al. 2011). We assume a fix dust-to-gas mass ratio of $1 \%$. We then use the dust model to compute the emission spectra of the three dust components (PAHs, VSGs, and BGs) illuminated by the incident radiation field from the star cluster NGC 6611.

We use the STARBURST99 online model ${ }^{3}$ described in Leitherer et al. (1999) and Vázquez \& Leitherer (2005) to define the spectral shape of the radiation field from the illuminating star cluster NGC 6611. We use the parameters of two million year

\footnotetext{
3 http://www.stsci.edu/science/starburst99/
}

old cluster, Salpeter initial mass function $\left(\mathrm{d} n / \mathrm{d} M \propto M^{-2.35}\right)$, and stellar masses from $1 M_{\odot}$ to $100 M_{\odot}$. The modeled radiation field corresponds to $1.6 \times 10^{9} L_{\odot}$, which we normalize to ensure agreement with the total flux of the most massive stars of the cluster. Dufton et al. (2006b) presented an analysis of VLT-FLAMES spectroscopy for NGC 6611. Their online catalogue (Dufton et al. 2006a) lists stars classified as earlier than B9. The 42 members of NGC 6611 have a combined total luminosity of $3.4 \times 10^{6} L_{\odot}$, which is a factor of 480 lower than the Starburst99 model output spectrum. We apply that correction factor to the model spectrum of the radiation field. In Habing units, which represents the integrated intensity of the solar neighborhood from 912 to $2000 \AA$ or $1.6 \times 10^{-3} \mathrm{erg} \mathrm{s}^{-1} \mathrm{~cm}^{-2}$, the cluster radiation field intensity is $\chi_{0} \simeq 4800$ at a distance of 3 parsecs (see Sect. 4.4 for a discussion of the spatial variations in the IRSF). In the following, we use this value as a reference for the dust model.

For the features within the shell ("blob", "shell border" and "reverse shell"), the use of a non-attenuated radiation field is acceptable since the UV optical depth is low. For the features within the PDRs ("pillar", "spire" and "shoulder"), we have to take into account the extinction of the radiation field by the ionized layer of gas and the PDR layer itself. We model this in a simple way by removing the Lyman continuum photons and with a far-UV extinction of 1 mag. This extinction reflects that the emission from PDRs comes from a range of depths in UV-dark clouds with a weighting proportional to the UV field. A more detailed study of the PDRs is beyond the scope of this paper.

\subsection{MIPS $24 \mu \mathrm{m}$ to MIPS $70 \mu \mathrm{m}$ ratio as a tracer of $\chi$}

We first use the dust model of Compiègne et al. (2011) to compute the MIPS $24 \mu \mathrm{m}$ to MIPS $70 \mu \mathrm{m}$ ratio of the dust emission for different dust-size distributions to show how it is related to $\chi$. Within this wavelength range, the PAH contribution to the emission is weak relative to that of VSGs and BGs. Therefore, we present the MIPS $24 \mu \mathrm{m}$ to MIPS $70 \mu \mathrm{m}$ ratio as a function of $\chi$ for three size distributions: VSGs only, BGs only, and a mixture of VSGs and BGs that matches their relative abundances in the diffuse high Galactic latitude medium (DHGL, Compiègne et al. 2011). Therefore, we take into account any dust evolutionary process that would destroy a specific grain size component. Figure 12 shows the results along with the MIPS $24 \mu \mathrm{m}$ to MIPS $70 \mu \mathrm{m}$ ratio measured for the Eagle Nebula structures, both within the shell and the PDRs. The differences between the set of modeled curves for the PDRs and that for the shell are insignificant. We first make no distinction while presenting them. We then discuss our results for the PDRs and shell structures independently.

For a given $\chi / \chi_{0}$, VSGs always have a higher MIPS24/MIPS70 as they are hotter than BGs. However, for $\chi / \chi_{0} \gtrsim 1.0$, MIPS24/MIPS70 is almost independent, to within a factor of a few, of the dust-size distribution. These values of $\chi$ correspond to the large values of the MIPS24/MIPS70 (>1). For $\chi / \chi_{0} \lesssim 1.0$, MIPS24/MIPS70 is significantly more dependent on the grain size distribution with difference up to almost two orders of magnitude. Likewise, for a given MIPS24/MIPS70, the required $\chi / \chi_{0}$ is always higher for BGs than VSGs. The difference is as small as a factor of a few for high values of MIPS24/MIPS70 and as high as almost two orders of magnitude for low values of MIPS24/MIPS70. Therefore, given MIPS24/MIPS70, the constraint on the intensity of the IRSF is stronger for higher values of $\chi$ and requires a better 
N. Flagey et al.: Tracing the energetics and evolution of dust with Spitzer: a chapter in the history of the Eagle Nebula

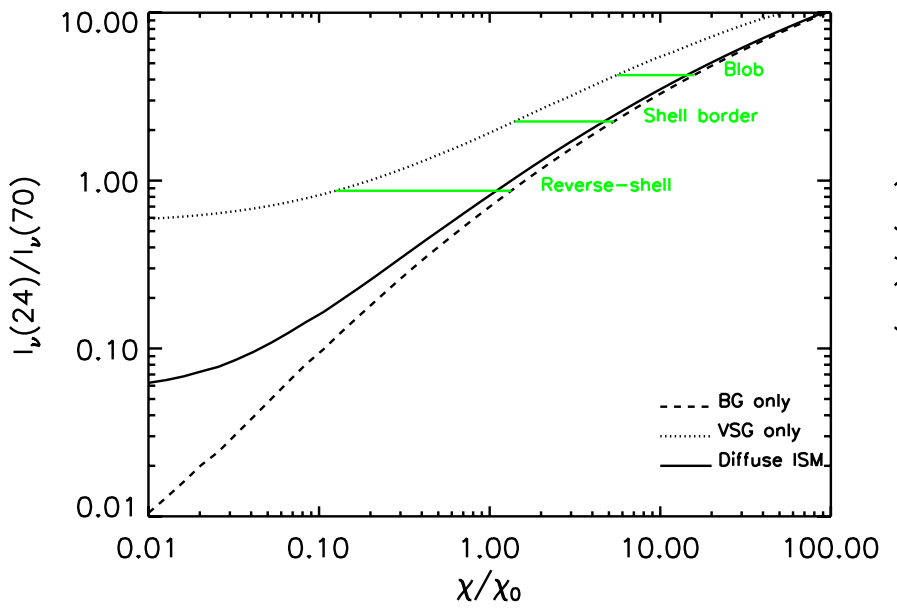

(a)

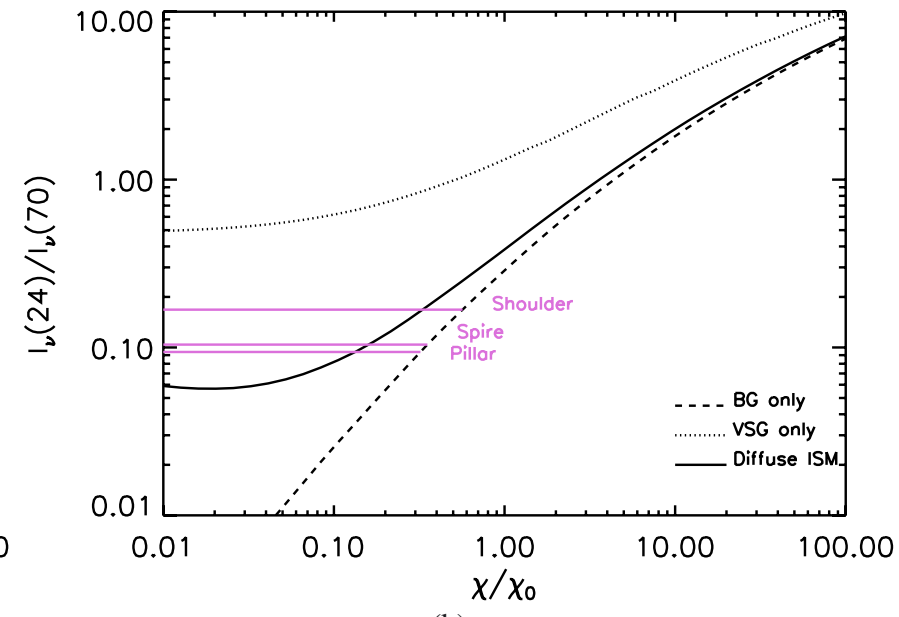

(b)

Fig. 12. MIPS $24 \mu \mathrm{m}$ to MIPS $70 \mu \mathrm{m}$ ratio as a function of the radiation field intensity, as predicted by the model of Compiègne et al. (2011). Several dust-size distribution are used: (dashed line) BGs only, (dotted-line) VSGs only, and (solid line) a mixture of BGs and VSGs. The MIPS24to-MIPS70 ratio for several structures within M 16 is indicated. The radiation field spectral shape is as described in the text with a) no extinction, or b) $\mathrm{A}(\mathrm{FUV})=1 \mathrm{mag}$ and the Lyman continuum photons removed.

Table 1. Lower and upper limits of $\chi / \chi_{0}$ for the whole set of structures as deduced from their MIPS $24 \mu \mathrm{m}$ to MIPS $70 \mu \mathrm{m}$ ratio.

\begin{tabular}{lccc}
\hline \hline Shell structure & $\chi / \chi_{0}$ & PDR structure & $\chi / \chi_{0}$ \\
\hline Reverse shell & $0.13-1.3$ & Pillar & $<0.3$ \\
Blob & $5.6-16$ & Shoulder & $<0.6$ \\
Shell border & $1.4-5.4$ & Spire & $<0.4$ \\
\hline
\end{tabular}

knowledge of the dust-size distribution (e.g. as provided by other IR observations, see next subsection) at low values of $\chi$. In contrast, constraining the dust-size distribution requires an a priori on $\chi$, constraint of that can be placed more easily at low values of $\chi$.

According to the model, the PDR structures ("pillar", "spire" and "shoulder") require a radiation field intensity of at most a factor two lower than the reference, and no lower limit can be estimated because we have no constraint on the dust-size distribution. However, if we assume that it does not depart significantly from that of the DHGL medium, the MIPS24 to MIPS70 ratio within PDRs are most accurately interpreted with $\chi / \chi_{0} \simeq 0.1$. The inner shell structures ("blob", "shell border", and "reverse shell") are more on the high end of the radiation field intensity. The "shell border" and the "blob" are in contrast most successfully interpreted with a $\chi / \chi_{0}$ of at least a few and up to 16 , whether the dust-size distribution is dominated by BGs or VSGs. The difference between the radiation field intensity that illuminates these two structures and the PDRs is thus at least an order of magnitude. The "reverse shell" position however is not strongly constrained and overlaps those of the PDR structures. At this position, if the dust-size distribution is dominated by VSGs, then $\chi / \chi_{0} \simeq 0.1$ while $\chi / \chi_{0} \sim 1$ if the BGs contribute the most to the dust-size distribution. The full range of required radiation field intensities for each structure is given in Table 1.

The MIPS $24 \mu \mathrm{m}$ to MIPS $70 \mu \mathrm{m}$ flux ratio also indirectly provides us with a measurement of the equilibrium dust temperature $T_{\text {eq }}$ of the largest dust particles. In Fig. 13, we plot the BG equilibrium temperature, provided by the dust model, as a function of $\chi$, for both the PDR and the shell structures, and for both types of large grains used in the model of Compiègne et al. (2011): LamC and aSil. For a given radiation field intensity $\chi / \chi_{0}$, we plot the upper and lower limits to the equilibrium temperatures of each grain type. The difference between both types of BG components is not really significant. In particular, the lower limits are almost identical. In Fig. 13, we hatch the range of equilibrium temperatures for the values of $\chi / \chi_{0}$ given by Fig. 12: $0.13<\chi / \chi_{0}<16$ for the Shell and $\chi / \chi_{0}<0.6$ for the PDR structures. While the smallest LamC grains in the PDR structures may reach equilibrium temperature as high as $71 \mathrm{~K}$, those are limited in number. Likewise, only the largest grains in the shell structures may reach equilibrium temperature as low as $24 \mathrm{~K}$. The majority of the grains, as traced by the most abundant size bin of each BG component (also plotted in Fig. 13), span a range of equilibrium temperatures that does not overlap significantly for the shell and the PDR structures. For the PDR structures, equilibrium temperatures for the most abundant size $20<T_{\text {eq }}<50 \mathrm{~K}$, while for the inner shell structures $35 \mathrm{~K}<T_{\text {eq }}<100 \mathrm{~K}$. Therefore, equilibrium temperatures above $50 \mathrm{~K}$ can only be efficiently reached by BGs in the inner shell, while equilibrium temperatures below $50 \mathrm{~K}$ are mostly found in the PDRs. The dust in the inner shell is thus significantly hotter than that in the PDRs. Indebetouw et al. (2007) used the IRAS $60 \mu \mathrm{m}$ to IRAS $100 \mu \mathrm{m}$ ratio to build a low spatial resolution $\left(4.3^{\prime}\right)$ color temperature map of the dust in M 16 . Their values range from $32 \mathrm{~K}$ in the molecular cloud to $40 \mathrm{~K}$ inside the nebula. We build the same map (not shown here) using the ratio of IRAS $25 \mu \mathrm{m}$ to IRAS $60 \mu \mathrm{m}$ flux (to better match the MIPS24 to MIPS $70 \mu \mathrm{m}$ diagnostic) and find a color temperature ranging from $45 \mathrm{~K}$ to $65 \mathrm{~K}$, which is in closer agreement with our measurements of the $\mathrm{BG}$ equilibrium temperature in the shell. The remaining difference may come from the lower spatial resolution that averages "hot" features with "cold" features within the beam.

\subsection{Fitting of the whole IR SED}

The additional measurement provided by MIPS $70 \mu \mathrm{m}$ enables us to place some constraint on the radiation field intensity that is required to heat the dust to the observed temperatures. Hereafter, 


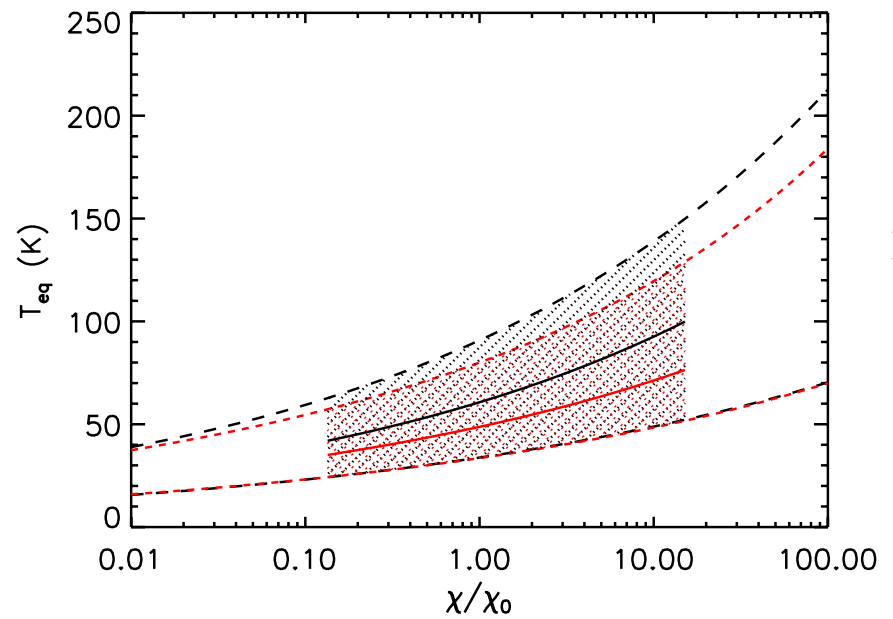

(a)

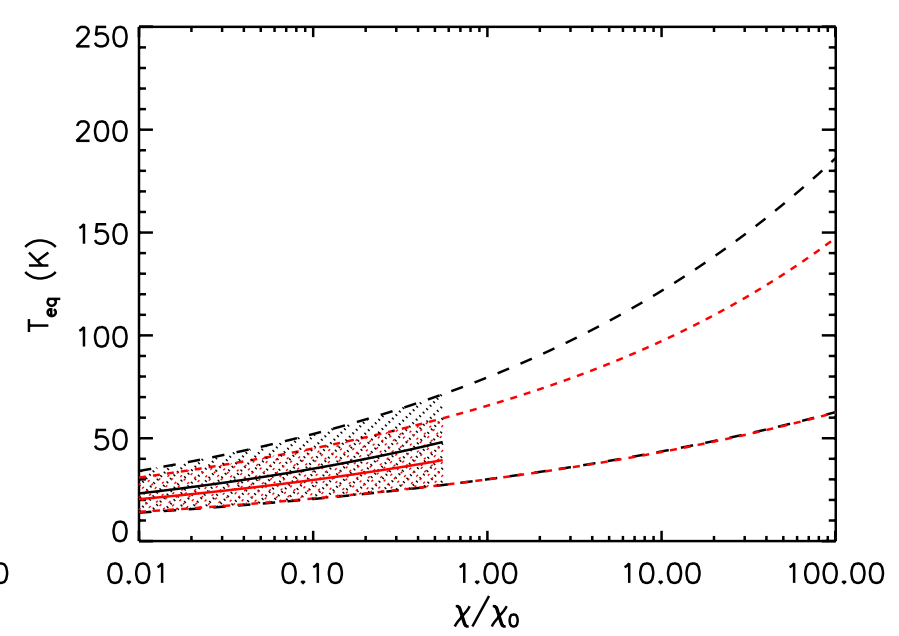

(b)

Fig. 13. BG equilibrium temperature as a function of the radiation field intensity. The hatched area corresponds to the range of equilibrium temperatures span by the entire BGs size distribution. The solid lines represent the equilibrium temperature for the most abundant size bin. The hatched area and the solid line are only plotted for the values of $\chi / \chi_{0}$ that are given by Fig. 12. Black is for LamC grains, red is for aSil grains as described in Compiègne et al. (2011). The radiation field spectral shape is that mention in the text with a) no extinction, b) $\mathrm{A}(\mathrm{FUV})=1 \mathrm{mag}$ and the Lyman continuum photons removed.

Table 2. Best-fit parameters for SEDs of the Eagle Nebula.

\begin{tabular}{lcccccc}
\hline \hline Position & $\begin{array}{c}I_{24} \\
(\mathrm{MJy} / \mathrm{sr})\end{array}$ & $\begin{array}{c}\chi / \chi_{0} \\
\chi / \chi_{0}\end{array}$ & $\begin{array}{c}Y_{\mathrm{PAH}} \\
\left(M / M_{\mathrm{H}}\right)\end{array}$ & $\begin{array}{c}Y_{\mathrm{VSG}} \\
\left(M / M_{\mathrm{H}}\right)\end{array}$ & $\begin{array}{c}Y_{\mathrm{BG}} \\
\left(M / M_{\mathrm{H}}\right)\end{array}$ & $\begin{array}{c}\sigma_{\text {dust }} \\
\left.(\mu \mathrm{g} \mathrm{cm})^{-2}\right)\end{array}$ \\
\hline DHGL & & & $7.8 \times 10^{-4}$ & $1.65 \times 10^{-4}$ & $9.25 \times 10^{-3}$ & 1.7 \\
Pillar & 240 & $0.19 \pm 0.04$ & $(2.64 \pm 0.57) \times 10^{-4}$ & $(2.45 \pm 0.90) \times 10^{-4}$ & $(9.49 \pm 1.82) \times 10^{-3}$ & 380 \\
Shoulder & 60 & $0.43 \pm 0.08$ & $(2.51 \pm 0.45) \times 10^{-4}$ & $(1.12 \pm 0.95) \times 10^{-4}$ & $(9.64 \pm 2.15) \times 10^{-3}$ & 33 \\
Spire & 250 & $0.12 \pm 0.05$ & $(2.96 \pm 1.27) \times 10^{-4}$ & $(5.09 \pm 2.89) \times 10^{-4}$ & $(9.20 \pm 3.62) \times 10^{-3}$ & 870 \\
Shell border & 230 & $4.36 \pm 1.36$ & $(4.85 \pm 1.12) \times 10^{-6}$ & $(3.69 \pm 2.71) \times 10^{-4}$ & $(9.63 \pm 2.77) \times 10^{-3}$ & 0.2 \\
Blob & 630 & $9.69 \pm 2.33$ & 0 & $(5.98 \pm 3.07) \times 10^{-4}$ & $(9.40 \pm 1.82) \times 10^{-3}$ & 2.9 \\
Reverse shell & 90 & $1.15 \pm 0.13$ & 0 & $(1.99 \pm 0.31) \times 10^{-3}$ & $(8.01 \pm 0.23) \times 10^{-3}$ & 2.1 \\
Shell Border & 230 & $2 *$ & $(6.68 \pm 4.47) \times 10^{-5}$ & $(1.05 \pm 0.77) \times 10^{-2}$ & $(3.59 \pm 2.29) \times 10^{-4}$ & 0.17 \\
$a_{0}$ (VSG) =5.5 nm & & & & & & \\
\hline
\end{tabular}

Notes. The radiation field intensity, the dust-size distribution, in terms of relative mass ratio abundances, and the total dust column density are given. The parameters for the diffuse high Galactic latitude (DHGL) reference of Compiègne et al. (2011) are also given. The dust-to-gas mass ratio is fixed at 0.01 therefore a dust mass column density of $1.7 \mu \mathrm{g} \mathrm{cm}^{-2}$ corresponds to $10^{20} \mathrm{H} \mathrm{cm}^{-2}$. The MIPS $24 \mu \mathrm{m}$ peak surface brightness is also given for each structure.

we use our dust model and the whole IR SED of each structure within M 16 to better determine the variation in $\chi$ and the dustsize distribution at the same time.

We set four parameters free: the intensity of the radiation field and the three mass abundances relative to $\mathrm{H}$ corresponding to the three dust components (PAH, VSG, and BG). The spectral shape of the radiation field is that described in Sect. 4.1. The other parameters describing the dust-size distribution (e.g. the size range and distribution shape) are those presented in Compiègne et al. (2011). We use the MPFIT package ${ }^{4}$ for IDL (Markwardt 2009) to constrain the free parameters, given the SED. We use the default tolerance parameters and limit the four parameters to positive values. The best-fit spectra are shown in Fig. 14 and the best-fit parameters are given in Table 2. We also estimate the dust column density for each feature assuming a dust-to-gas mass ratio of 0.01 .

The positions within the PDRs are best fit with low values of $\chi / \chi_{0}\left(\right.$ a few $\left.10^{-1}\right)$ in agreement with the upper limits from

\footnotetext{
$\overline{{ }^{4} \text { http://purl.com/net/mpfit }}$
}

Table 1, a factor of a few less PAHs and a factor of a few more or less VSGs than in the DHGL medium. An increase/decrease in the small grains abundance by a factor of a few within PDRs of NGC $2023 \mathrm{~N}$ and the Horsehead Nebula has already been observed by Compiègne et al. (2008) and within translucent sections of the Taurus Molecular Complex by Flagey et al. (2009b). The low values of $\chi / \chi_{0}$ required to fit the SED of the PDRs can partly be explained with shadow effects within the nebula. Another parameter that we do not take into account in our simple model is the geometry of the features and the resulting limb brightening effect. The dust column density for the "pillar" and the "spire" position is indeed about a few $10^{-4} \mathrm{~g} \mathrm{~cm}^{-2}$, which corresponds to a gas column density of a few $10^{22} \mathrm{~cm}^{-2}$ or a visual extinction of a few magnitudes, significantly larger than that required to attenuate the incident UV radiation field. The three "PDR" positions give very similar results, especially in terms of PAH abundance which varies by less than $10 \%$. The VSG abundance varies more significantly, by up to a factor of five. The BG always dominates the dust-size distribution with abundances very close to that of the DHGL. 


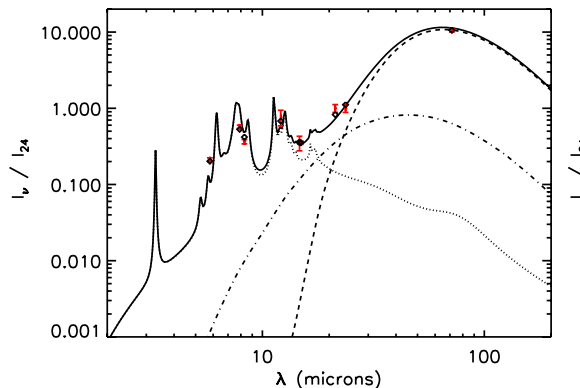

(a) Pillar

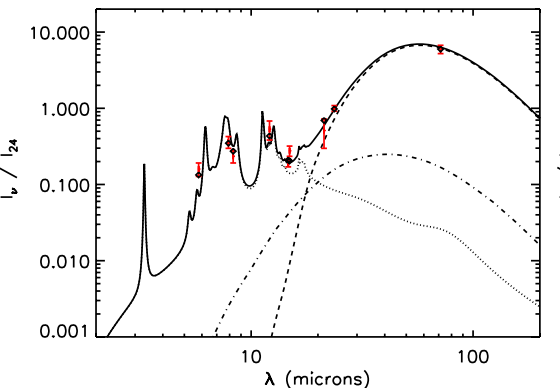

(b) Shoulder

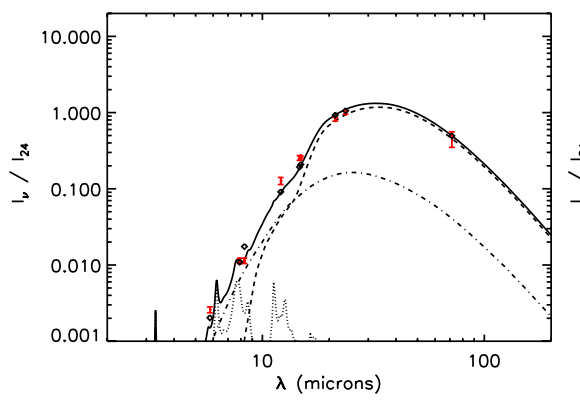

(d) Shell border

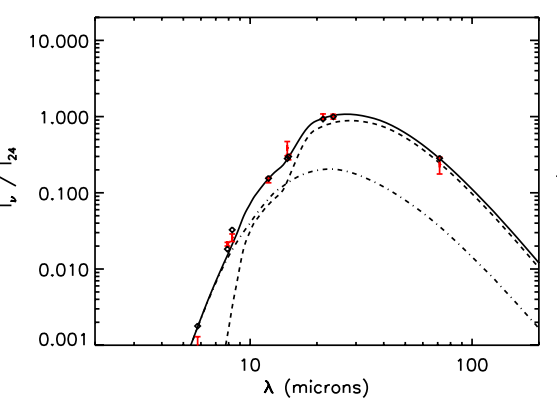

(e) Blob

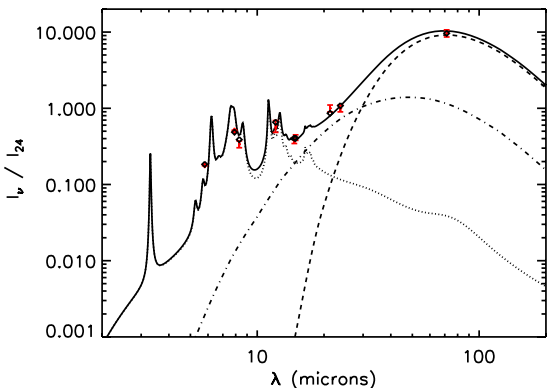

(c) Spire

Fig. 14. Best-fit for a) the "pillar", b) the "shoulder", c) the "spire", d) the "shell border", e) the "blob", f) the "reverse shell". In each panel, the solid line is the total model spectrum, the dotted line, the dash-dotted line and the dashed line represent, respectively, the PAH, the VSG, and the BG contributions. Diamonds are model broadband fluxes. Red crosses are measurements.

The positions within the "shell" require larger values of $\chi / \chi_{0}$ (about a few) in agreement with values from Table 1, a significant depletion of the PAHs and a significant increase in the VSG abundance, up to a factor 10, with respect to the PDR values. The total dust column density is about $\sim 10^{-6} \mathrm{~g} / \mathrm{cm}^{2}$, which is similar to DHGL values and corresponds to a gas column density of about $10^{20} \mathrm{~cm}^{-2}$. As a consequence of the increased $\chi$, the VSG and BG emission spectra peak at almost identical wavelengths (see Figs. 14d-f). We show in the previous section that MIPS24/MIPS70 is a good tracer of $\chi$ but not of the dust-size distribution, especially at high values of $\chi$. Here, the addition of the other IR observations provides stronger constraints on the VSG and BG abundances. For the position of the "reverse shell", the initial best-fit model (not shown here) underestimates the MIPS $70 \mu \mathrm{m}$ measurement by almost an order of magnitude. As a consequence, the required $\chi / \chi_{0}$ is overestimated relative to that from Table 1 derived from the MIPS24 to MIPS70 ratio. We believe this poor fit at the longer wavelengths is due to the uneven number of measurements at short to long wavelengths, relative to the peak of the dust emission. From 6 to $24 \mu \mathrm{m}$, no fewer than seven measurements are available, while only MIPS $70 \mu \mathrm{m}$ data is available for wavelengths longer than the peak position. The fit process is thus biased towards shorter wavelengths. To limit this effect, we repeat the fitting process of the "reverse shell" position with an increased weight on the MIPS $70 \mu$ m measurement. Figure 14f shows the result of that fit. The three positions within the shell give results that are very similar to each other and very different from those of the PDR positions: (1) an incident radiation field intensity a factor of a few larger than that provided by the star cluster NGC 6611 and about an order of magnitude larger than that required for the PDR positions; (2) a significant depletion of the PAHs; and (3) an increase in the VSG abundance relative to that of the BGs.
To explore the importance of a change in the dust-size distribution, we redo the fit of the "shell border" with a fixed intensity of the radiation field $\chi / \chi_{0}=2$ and a free mean size of the VSG component $\left(a_{0}\right)$. In the model of Compiègne et al. (2011) for the DHGL medium, the VSG size distribution is assumed to have a log-normal distribution (with the center radius $a_{0}=2 \mathrm{~nm}$ and the width of the distribution $\sigma=0.35 \mathrm{~nm}$ ). We keep the width of the log-normal distribution constant and allow the center size $a_{0}$ to vary between 0.6 and $20 \mathrm{~nm}$. The other free parameters for that fit are the abundances of the dust components, as previously. The best-fit model is plotted in Fig. 15 and the parameters are given in Table 2. A significant increase in the mean size of the VSGs relative to that in the DHGL medium, by almost a factor of 3, is required. There are almost no PAHs, as in the previous fits. The BGs are about a factor of 3 less abundant than in the previous fit and about a factor of 30 less abundant than in the DHGL. The abundance of the VSGs is about 60 times higher than in the DHGL medium, though the uncertainty remains large $(\sim 75 \%)$. Therefore, the "shell border" SED requires that most of the dust mass is concentrated into the VSG component. Despite these variations in the dust-size distribution, the total dust column density remains very similar to that of the fit with a fixed mean size for VSGs $\left(0.17\right.$ instead of $\left.0.20 \mu \mathrm{g} \mathrm{cm}^{-2}\right)$. We also try the same fit with $\chi / \chi_{0}=1$ but find that the uncertainties in the parameters are then significantly higher $(>100 \%)$.

We conclude that the MIR shell SED can be accounted for either by means of a significant change in the dust-size distribution or an additional source of heating besides the star cluster radiation field. In the following, we first discuss two sources of UV heating that may account for the values of $\chi / \chi_{0}>1$ required to fit the "Shell" SEDs. The first one is related to the spatial variations in $\chi$ caused by the exact positions of the OB stars in the sky. The second originates in the Lyman $\alpha$ photons emitted by the hydrogen and absorbed by the dust grains. We then 


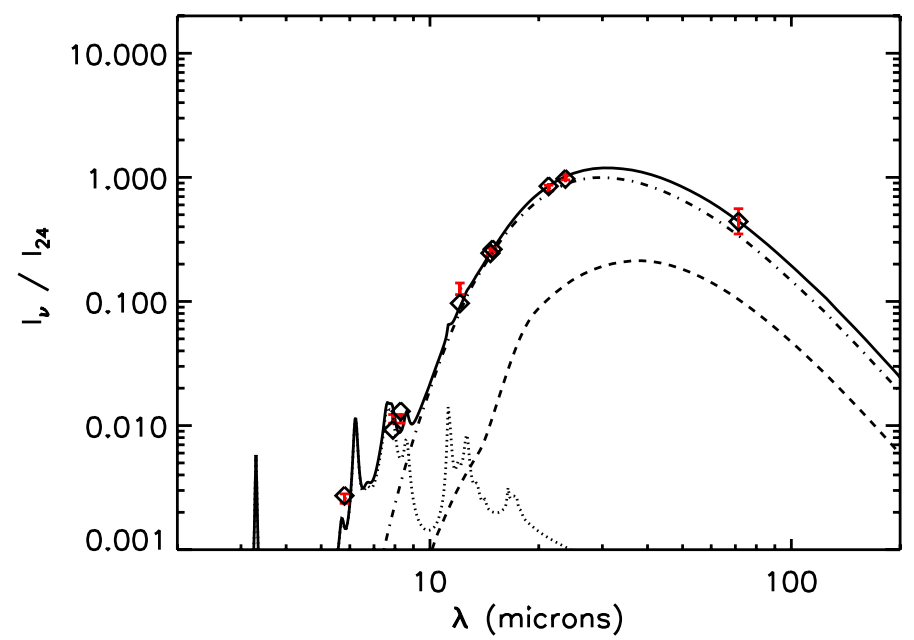

Fig. 15. Same as Fig. 14d but with $\chi / \chi_{0}=2$ and a free mean size of the VSG component. The solid line is the total model spectrum, the dotted line, the dash-dotted line and the dashed line represent, respectively, the PAH, the VSG, and the BG contributions. Diamonds are model broadband fluxes. Red crosses are measurements.

Table 3. Correction factors on $\chi_{0}$ from the dispersion of the stars in the sky plane and corrected $\chi / \chi_{0}$ required for the best-fit solutions.

\begin{tabular}{lcc}
\hline \hline Position & $\begin{array}{c}\text { Correction } \\
\text { factor }\end{array}$ & $\begin{array}{c}\text { Corrected } \chi / \chi_{0} \\
\text { (best fit) }\end{array}$ \\
\hline Shell border & $<1.5$ & $>2.9$ \\
Blob & $<4.5$ & $>2.1$ \\
Reverse shell & $<6.8$ & $>0.2$ \\
\hline
\end{tabular}

consider, in the next section, another heating process originating from collisions with the gas.

\subsection{Spatial variations in the incident radiation field}

Depending on the exact positions of the main OB stars of NGC 6611 within the Eagle Nebula, the local incident radiation field intensity may vary and thus explain the required values of $\chi / \chi_{0}$. For the "cold" PDRs features, it is easy to explain values of $\chi / \chi_{0}<1$ as the stars are not altogether on the plane of the sky, in addition to the probable shadow effects already mentioned. However, the required values of $\chi / \chi_{0}>1$ for the "shell" structures cannot be accounted for by the same interpretation. In Fig. 1, we indicate the position and the spectral type of the members of NGC 6611, according to Dufton et al. (2006a). We compute the variations in the radiation field intensity $\chi_{0}$ as a function of the position, taking into account the luminosity and position of each individual member of the cluster. We assume that all the stars and the "shell" structures are in the same plane of the sky. Therefore, the values of the local radiation field intensity we compute are thus upper-limits and the corrected values of $\chi / \chi_{0}$ required for the best-fits models are lower limits. All these values are reported in Table 3 . The corrections factors are about a factor of a few at most. The required values of $\chi / \chi_{0}$ for the "shell border" and the "blob" are still at least a factor 2 to 3 higher than that provided by the star cluster.

The position of the members of NGC 6611 also reveals that Pilbratt's Blob is very close to an 08.5V star, as shown in Fig. 16. This suggests a possible local action of the winds from this star. The shock provided by the winds may account for a local enhancement in the density within the shell and possibly for dust

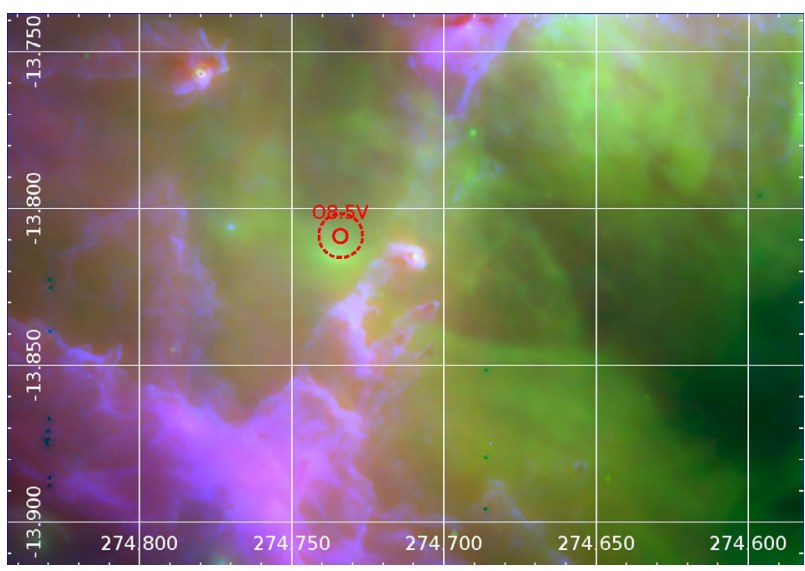

Fig. 16. Zoomed image of the three color image at the position of the Blob. The position and spectral type of the nearest $\mathrm{O}$ star is also reported. The red dashed circle, centered on the $08.5 \mathrm{~V}$ star has a 26 arcsec radius (about $0.25 \mathrm{pc}$ at the distance of $\mathrm{M} 16$ ).

processing. The same interpretation does not hold for the "shell border" and the "reverse shell" position, which are both very distant from any OB star, as also shown in Fig. 16. We discuss collisional heating in Sect. 5.

\subsection{Lyman alpha photon heating}

We show here that Lyman $\alpha$ photons are not a significant heat source for the shell. Every Lyman $\alpha$ photons emitted by an hydrogen atom, after multiple absorption and reemission by other hydrogen atoms, either succeeds in escaping the medium or is absorbed by a dust grain. The Lyman $\alpha$ contribution to the dust IR brightness is $S_{\mathrm{Ly} \alpha}=\int n_{\mathrm{e}} \times n_{\mathrm{H}^{+}} \times a_{2} \times h v_{\mathrm{Ly} \alpha} \mathrm{d} l=$ $E M \times a_{2} \times h v_{\mathrm{Ly} \alpha}$, where $E M$ is the emission measure and $a_{2}$ the hydrogen recombination coefficient to levels 2 and higher. The equation assumes that all recombinations from excited levels produce a Ly $\alpha$ photon that is absorbed by dust.

We compute the $E M$ from $\mathrm{Br} \gamma$ observations of M 16 obtained at the Canada-France-Hawaii Telescope (CFHT). These observations will be presented in a future paper. They do not show a counterpart of the "blob", but there is an increase in the $\operatorname{Br} \gamma$ emission associated with the "shell border" of $E M=$ $3.5 \times 10^{3} \mathrm{pc} \mathrm{cm}^{-6}$. The Ly $\alpha$ photon total flux that we estimate from these measurements is $S_{\mathrm{Ly} \alpha}=0.048 \mathrm{erg} \mathrm{s}^{-1} \mathrm{~cm}^{-2}$. In comparison, the $24 \mu \mathrm{m}$ brightness of the "shell border" is $230 \mathrm{MJy} / \mathrm{sr}$, which corresponds to a bolometric intensity of $0.37 \mathrm{erg} \mathrm{s}^{-1} \mathrm{~cm}^{-2}$ that we measure from the best-fit solution (see Fig. 7b) between 1 and $1000 \mu \mathrm{m}$. The extra heating provided by the Ly $\alpha$ photons is thus about a factor of 8 too small.

\section{Collisional heating of dust}

We face the difficulty of explaining the shell infrared colors with UV heating by considering the possibility that gas-grains collisions provide additional dust heating. We quantify the conditions that would be required to fit the shell SED with a combination of radiative + collisional heating of dust.

We use the work of Dwek (1987) to quantify the heat deposited in the grain by collisions with electrons as a function of grain size and plasma temperature. As in Sect. 4, we use the DUSTEM model with a combination of silicate and amorphous carbon grains (Compiègne et al. 2011). Since the DUSTEM code 
does not include collisional excitation, we wrote a specific module to compute the distribution of grain temperatures for stochastic heating by both photons and collisions. This code takes into account the Maxwellian distribution of the electron kinetic energy. The results of our calculations are illustrated in Fig. 17 for carbon grains. The Spitzer colors $I_{v}(8 \mu \mathrm{m}) / I_{\nu}(24 \mu \mathrm{m})$ and $I_{\nu}(24 \mu \mathrm{m}) / I_{\nu}(70 \mu \mathrm{m})$ are plotted versus grain size for radiative heating by the mean Eagle Nebula radiation field, and radiative + collisional heating for a range of electron densities $n_{\mathrm{e}}$. The temperature of the electrons $T_{\mathrm{e}}$ is fixed to $10^{6} \mathrm{~K}$. Our specific choice of $T_{\mathrm{e}}$ is not critical, because the colors depend mainly on the plasma pressure, i.e., the product $n_{\mathrm{e}} \times T_{\mathrm{e}}$. Collisional heating has a significant impact on the infrared colors for pressures $p / k$ higher than a few $10^{7} \mathrm{~K} \mathrm{~cm}^{-3}$. The figure shows that both colors may be fit for pressures $p / k=1.9 \times n_{\mathrm{e}} T_{\mathrm{e}} \sim 5 \times 10^{7} \mathrm{~K} \mathrm{~cm}^{-3}$ and a characteristic grain size of $\sim 10 \mathrm{~nm}$. For this plasma pressure, collisions with electrons dominate the heating of small grains with radii $<10 \mathrm{~nm}$, while radiation is the main heating source for larger grains. To illustrate the ability of the dust model to fit the shell SED, we use a dust-size distribution that combines a log-normal size distribution for very small carbon grains plus a power-law size distribution for silicates. We keep the relative fractions of dust mass in carbon grains and silicates to their interstellar values of $1 / 3$ and $2 / 3$, respectively. In Fig. 18, we show a fit of the "shell border" SED obtained for $n_{\mathrm{e}}=30 \mathrm{~cm}^{-3}$ and $T_{\mathrm{e}}=10^{6} \mathrm{~K}$. For this fit, the characteristic radius (i.e. the mean value of the log-normal size distribution) of the carbon VSGs is $6.5 \mathrm{~nm}$. This value is somewhat smaller than the one that can be inferred from Fig. 17, because the silicates contribute to about half of the $70 \mu \mathrm{m}$ flux. The figure shows that for a given plasma temperature the characteristic grain size is tightly constrained by the $I_{v}(8 \mu \mathrm{m}) / I_{v}(24 \mu \mathrm{m})$ ratio. It depends on the plasma temperature because this constraint is related to the stochastic heating of the smallest grains by collisions with electrons. The model also allows us to estimate the dust mass in the shell. The dust surface density is $2 \times 10^{-3} M_{\odot} \mathrm{pc}^{-2}$. Scaling this value by the full extent of the shell (4 pc radius), we find a total dust mass of $3 \times 10^{-2} M_{\odot}$.

The pressure inferred from the modeling of the collisional heating may be compared with independent constraints on the pressure within the Eagle nebula. This comparison highlights the difficulties in adopting, but does not fully rule out, the collisional heating solution. The gas pressure inferred from Hubble observations of the optical line emission from the faint end of the photo-evaporation flows arising from pillar I is $p / k \sim$ $10^{7} \mathrm{~K} \mathrm{~cm}^{-3}$ (see Fig. 7b, abscissa 0 in Hester et al. 1996). This value sets an upper limit on the ambient pressure around the flows, which is lower than the pressure required for the collisional heating solution. One possible way out of this problem is that pillar I is not embedded in the shell. The shell pressure can also be estimated from Pilbratt's blob. The blob is close to an $08.5 \mathrm{~V}$ star known to be associated with the ionizing cluster of the Eagle Nebula (see Fig. 16). Its morphology and position on one side of the star suggests that it traces a bow shock created by a supersonic motion between the shell and the star (van Buren et al. 1990). If this interpretation is right, it places a constraint on the shell pressure. At the standoff distance $d_{\mathrm{o}}$, i.e. the distance between the star and the edge of the blob, there is a pressure equilibrium between the wind pressure and the ambient pressure plus the ram pressure associated with the star motion. Hence, the wind pressure at the standoff distance, $p_{\mathrm{w}}=\dot{M}_{\mathrm{w}} \times V_{\mathrm{w}} /\left(4 \pi \times d_{\mathrm{o}}^{2}\right)$, is an upper limit to the ambient pressure. From the $24 \mu \mathrm{m}$ image, $d_{\mathrm{o}}=0.2 \mathrm{pc}$. We use the empirical relation between wind momentum and stellar luminosity (Kudritzki \& Puls 2000) to infer that for an O8.5V star

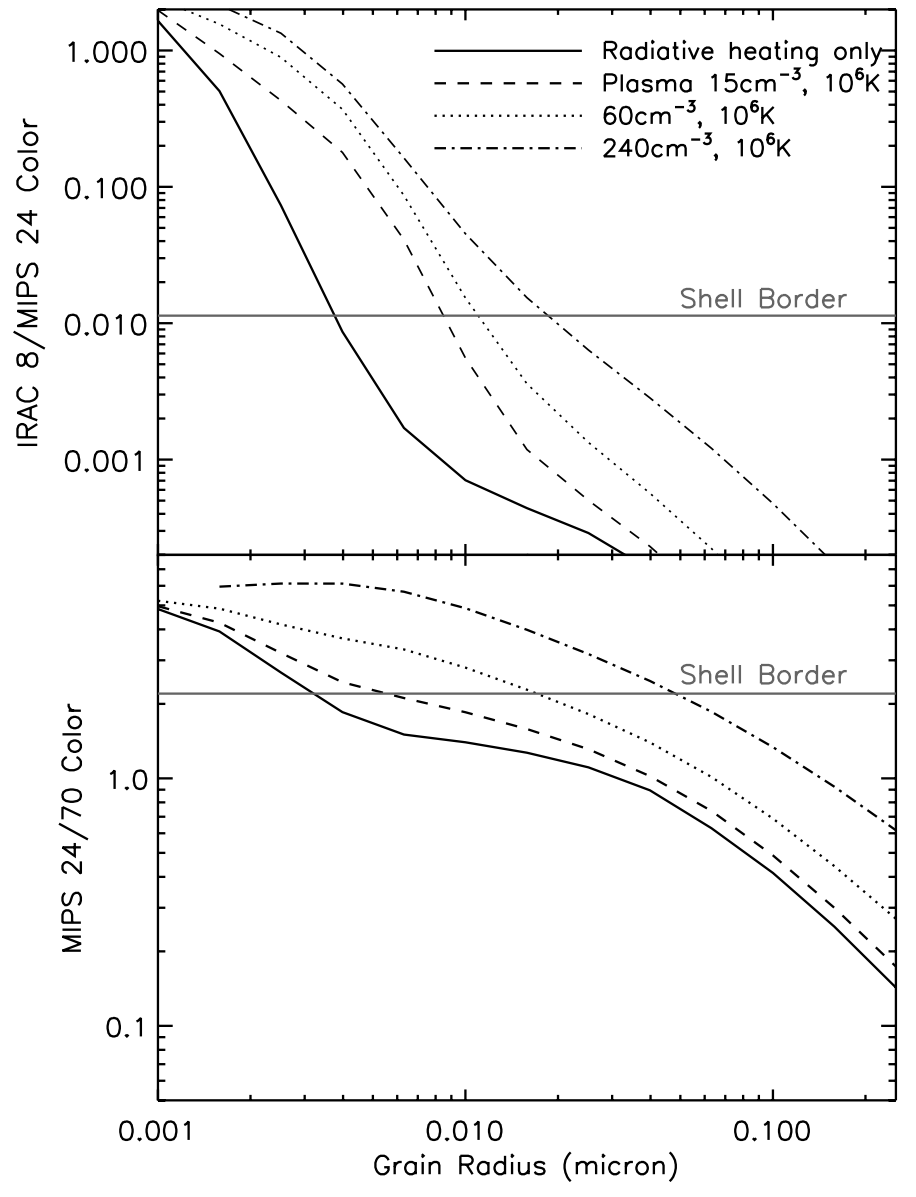

Fig. 17. Spitzer colors $I_{v}(8 \mu \mathrm{m}) / I_{v}(24 \mu \mathrm{m})$ and $I_{v}(24 \mu \mathrm{m}) / I_{v}(70 \mu \mathrm{m})$ for carbon grains versus grain size. The solid line give the colors for radiative heating for the Eagle Nebula radiation field. The other lines show the impact of collisional heating for a range of plasma pressure and a fixed temperature $T_{\mathrm{e}}$ of $10^{6} \mathrm{~K}$. A good fit of the shell SED is obtained for $n_{\mathrm{e}} \times T_{\mathrm{e}} \sim 3 \times 10^{7} \mathrm{~K} \mathrm{~cm}^{-3}$ (see Fig. 18).

$\dot{M}_{\mathrm{w}} \times V_{\mathrm{w}} \sim 2 \times 10^{-7} M_{\odot} \mathrm{yr}^{-1} \times 10^{3} \mathrm{~km} \mathrm{~s}^{-1}$. Hence, we find $p_{\mathrm{w}} / k=2 \times 10^{6} \mathrm{~K} \mathrm{~cm}^{-3}$, a value more than one order of magnitude smaller than the pressure required for the collisional heating solution. Here, the plausible way out would be that Pilbratt's blob is not a bow-shock.

\section{The nature of the mid-IR shell}

In this final part of the paper, we discuss the results of our dust modeling in the context of the Eagle Nebula massive-star forming region. We have shown in the previous sections that the dust SED of the MIR shell cannot be accounted for by standard models (i.e. interstellar dust heated by UV radiation). We find two possible explanations: (1) the fraction of the dust mass in stochastically heated VSGs is much larger in the shell than in the diffuse interstellar medium; (2) there is an additional source of heating which could be collisional heating in a high pressure plasma. Here we present two scenarios that can explain either or both of these requirements. In the first one, the mid-IR shell is a windblown shell, where the dust is heated by UV photons and where large grains have been ground into stochastically heated small particles. In the second scenario, we investigate a more speculative hypothesis where the shell would be a supernova remnant that would cool by means of IR dust emission. 


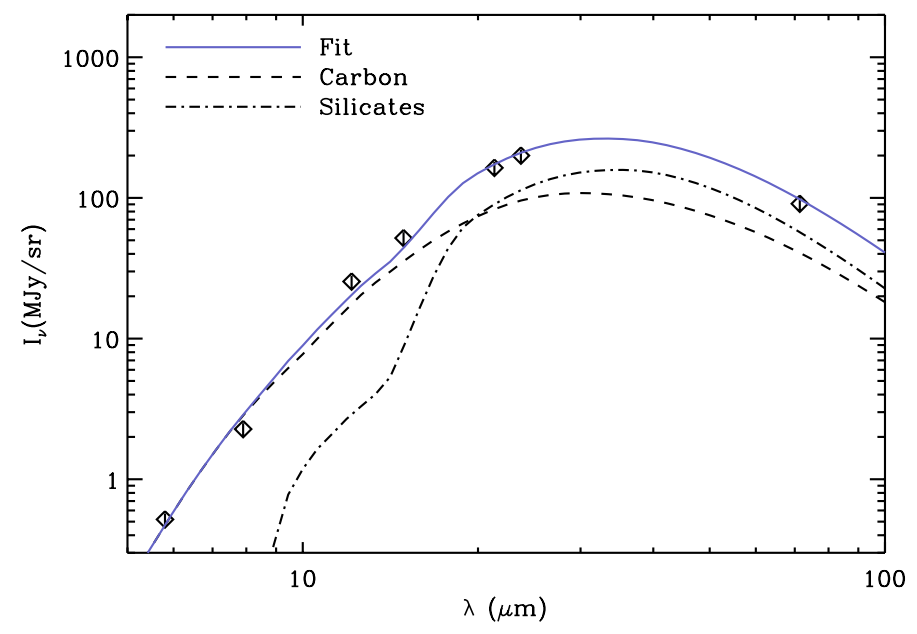

Fig. 18. Fit of the SED measured on the Eagle shell border with radiative plus collisional heating. The radiation field is that determined in Sect. 4 with $\chi / \chi_{0}=1$. The electron density is $30 \mathrm{~cm}^{-3}$ and the plasma temperature $10^{6} \mathrm{~K}$.

\subsection{A wind blown shell}

In this first scenario, matter outflowing from dense condensations and exposed to ionizing radiation from the stellar cluster, in particular the Eagle pillars, supply the shell with a continuous inflow of gas and dust. The mechanical pressure from the stellar winds pushes this matter outward, but the shell persists provided that its outward expansion is compensated for by continuing photo-evaporation. Since the shell is within the ionizing boundary of the nebula, the diffuse matter in the shell is fully ionized. The gas density and column density are too small to absorb all of the ionizing radiation. To quantify this scenario, we apply the empirical relation between wind momentum and stellar luminosity (Kudritzki \& Puls 2000) to each of the O stars in the cluster. For a shell inner radius of $3 \mathrm{pc}$, we find that the winds pressure is $p_{\text {winds }} / k=5 \times 10^{5} \mathrm{~K} \mathrm{~cm}^{-3}$. This value is a few times larger than the radiation pressure estimated from the shell infrared brightness $p_{\mathrm{rad}} / k \sim B_{\mathrm{IR}} / c \sim 10^{5} \mathrm{~K} \mathrm{~cm}^{-3}$, where $B_{\mathrm{IR}}$ is the mean bolometric IR brightness $\sim 0.4 \mathrm{erg} \mathrm{cm}^{-2} \mathrm{~s}^{-1}$ and $c$ the speed of light. The shell matter moves outward, because the wind pressure is higher than the average pressure in the interstellar medium. The expansion velocity is commensurate with the sound speed in the shell, and thus must be $\sim 10 \mathrm{~km} \mathrm{~s}^{-1}$. Since the shell is a few parsecs wide, the shell matter needs to be renewed over a timescale of a few $10^{5} \mathrm{yr}$ by on-going photo-evaporation.

In the Eagle Nebula, the pressure from stellar winds is too low to account for the shell colors with collisional excitation (see Sect. 5 for details). The mechanical power from the winds is also too small to contribute to the IR luminosity from the shell. For a wind velocity of $2500 \mathrm{~km} \mathrm{~s}^{-1}$ (Kudritzki \& Puls 2000), the mechanical energy injection is $\sim 2500 L_{\odot}$, a factor 20 smaller than the shell luminosity $\sim 5 \times 10^{4} L_{\odot}$ as estimated from the shell brightness $B_{\mathrm{IR}}$ and its angular diameter $\left(14^{\prime}\right)$. Unlike what Everett \& Churchwell (2010) advocated for N49, in M 16 the shell IR emission cannot be powered by the stellar winds, and does not represent a major cooling channel that impacts the dynamical evolution of a wind-blown shell.

The shell must originate from the only available source of dust, i.e., evaporating dense gas condensations within the ionization boundary of the Nebula. The difficulty in being certain that this is the right interpretation comes from interstellar dust (see Sects. 4 and 5). Our dust modeling in Sect. 4 indeed shows that the shell SED cannot be fit with the standard interstellar dust-size distribution. The fits shown in Figs. 14d and 15 illustrate the uncertainty in the modeling. It is beyond the scope of this paper to explore in a systematic way the full range of possible solutions, but we are confident that any fit will involve shattering of dust grains to nanometric sizes.

As a consequence of this interpretation of the Eagle Nebula shell, we conclude that massive-star forming regions have a major impact on carbon dust. Galliano et al. (2003) reached a similar conclusion in their modeling of the infrared SED of the dwarf, star-forming, galaxy NGC 1569. Observations of the ionized gas kinematics provide evidence of supersonic velocities in the immediate environment of pillars in star-forming regions (Westmoquette et al. 2009). Hence, the grinding of the carbon dust could be the result of grain shattering in grain-grain collisions within shocks driven by the dynamical interaction between the stellar winds and the shell. Theoretical modeling of the dust dynamics in shocks suggest that this is a plausible hypothesis (Jones 2004). Guillet et al. (2009) quantified dust processing by the passage of J-shocks of a few $10 \mathrm{~km} \mathrm{~s}^{-1}$. They find that the mass fraction in the largest grains is reduced to the profit of the smallest, as a result of grain shattering and dust vaporization.

\subsection{A supernova remnant}

Alternatively, we keep the usual distribution of dust grain sizes, but search for another source of pressure: a supernova remnant. This is not unexpected for a 3-Myr old nebula with very massive stars $\left(M_{\star} \sim 80 M_{\odot}\right.$ Hillenbrand et al. 1993). If so, we would be witnessing a specific time in the evolution of the remnant where the plasma pressure and temperature would be such that the remnant cools through dust emission. This scenario relates directly to the fit of the shell SED quantified in Sect. 5.

The infrared dust emission from fast shocks driven by supernovae has been quantified in several theoretical papers (e.g. Draine 1981; Dwek et al. 1996). Overall, dust is found to be a significant but not predominant coolant of shocked plasma because of dust destruction. For a dust to hydrogen mass ratio of $1 \%$ and a solar metallicity, dust cooling is larger than atomic cooling for temperatures $>5 \times 10^{5} \mathrm{~K}$, but, for temperatures $T$ higher than $\sim 10^{6} \mathrm{~K}$, the dust destruction timescale by sputtering is shorter than the gas cooling time (Smith et al. 1996; Guillard et al. 2009). This framework has been used to interpret observations of young remnants starting from the first infrared detections of supernovae with the IRAS survey (Dwek 1987). We propose here a distinct idea, where the shell infrared emission seen towards the Eagle Nebula would be related to the late evolution of a remnant.

For the model shown in Fig. 18, one-third of the shell infrared emission is powered by grain collisions with electrons and contributes to the plasma cooling. The remaining two-thirds is provided by radiative heating of the dust. Assuming that the dust infrared emission is the dominant gas cooling channel, the isobaric cooling time of the infrared emitting plasma is $t_{\text {cool }}=\frac{5}{2} \times 2.3 \times k T_{\mathrm{e}} /\left(\Gamma \times m_{\mathrm{p}} \times x_{\mathrm{d}}\right)$, where $\Gamma$ is the collisional heating rate per unit dust mass, $m_{\mathrm{p}}$ the proton mass and $x_{\mathrm{d}}$ the dust to hydrogen mass ratio. With the $\Gamma$ value derived from the fit in Fig. 18, we find that $t_{\text {cool }}=1500 \times\left(x_{\mathrm{d}} / 0.01\right)^{-1} \mathrm{yr}$. The dust-to-hydrogen mass ratio $x_{\mathrm{d}}$ is not constrained by the modeling. This factor may well be smaller than the reference value of $1 \%$ related to dust destruction by sputtering. The SED fit also allows us to estimate the plasma column density and thereby the internal energy $U$ of the infrared emitting plasma. The model gives $N_{\mathrm{H}}=8 \times 10^{18} \times\left(x_{\mathrm{d}} / 0.01\right)^{-1} \mathrm{H} \mathrm{cm}^{-2}$. From there, we 
find that $U=2 \times 10^{48} \times\left(x_{\mathrm{d}} / 0.01\right)^{-1} \mathrm{erg}$. This value is a small fraction of the expansion energy associated with a typical supernova explosion $\left(\sim 10^{51} \mathrm{erg}\right)$. Within our remnant hypothesis, this large difference indicates that the cooling time is short and that only a small fraction of the shocked plasma is contributing to the infrared emission. One possible explanation of this would be that we are observing the late evolution of the remnant when the low-density hot plasma heated to high temperatures early in the expansion of the remnant is cooling through turbulent mixing with photo-ionized gas (Begelman \& Fabian 1990). This plasma would have a long intrinsic cooling timescale, because its dust would have been destroyed early in the evolution of the remnant. For a pressure of $p / k=5 \times 10^{7} \mathrm{~K} \mathrm{~cm}^{-3}$, the cooling timescale through atomic processes of a dust-free plasma at a temperature of $10^{7} \mathrm{~K}$ is $2 \times 10^{6} \mathrm{yr}$.

This interpretation will need to be tested against additional observations. The absence of bright diffuse emission in the Chandra X-ray images (Linsky et al. 2007) can possibly be accounted for. For instance, the hot plasma may be too tenuous to be seen in emission, while the X-ray emission from the turbulent mixing layers would be soft and thus heavily attenuated by foreground gas. We re-analyzed the Chandra ACIS-I observations of M 16 (Linsky et al. 2007) to search for a faint background emission. After removal of point sources, we do find residual X-ray emission over the SW section of the mid-IR shell where the foreground extinction is the lowest. The emission spectrum fit gives $k T$ in the range $0.6-2 \mathrm{keV}$ and a foreground column density within $2.4-5.4 \times 10^{22} \mathrm{H} \mathrm{cm}^{2}$. The absorption-corrected $\mathrm{X}$-ray brightness is $1.3 \times 10^{3} \mathrm{erg} \mathrm{s}^{-1} \mathrm{~cm}^{2} \mathrm{sr}^{1}$. If this emission arises from the mid-IR shell (i.e. from a sightline length $\sim 10 \mathrm{pc}$ ), we derive a plasma pressure $p / k \sim 10^{8} \mathrm{~K} \mathrm{~cm}^{3}$. This result does not allow us to conclude that the $\mathrm{X}$-ray emission arises from a supernova remnant, but, if it does, the X-ray emission is consistent with the dust being collisionally excited in a high pressure plasma. In this case, if the X-ray emission filled the mid-IR cavity, the shell X-ray luminosity would be $\sim 10^{33} \mathrm{erg} \mathrm{s}^{-1}$. This is on the low side for an SNR: for comparison, the W28 SNR, which is interacting with a molecular cloud, has a total X-ray luminosity $L_{X} \sim 6 \times 10^{34} \mathrm{erg} \mathrm{s}^{-1}$ (Rho \& Borkowski 2002). However, our value of $L_{X}$ for the putative M 16 SNR is a lower limit, since it does not take into account the soft X-ray emission from cooler gas that is more heavily absorbed. Further X-ray observations are planned to clarify this point. MIR spectroscopic maps of M 16 with Spitzer, covering a wide range of emission features and ionization energies, will provide an additional test of this interpretation.

\section{Conclusions}

We have presented new IR images of the Eagle Nebula from the MIPSGAL survey that reveal the well-known illuminated clouds of dust and gas. The MIPS $24 \mu \mathrm{m}$ observations shows the same inner shell-like feature as mid-infrared observations from ISO or MSX. It is significantly brighter than the PDRs. Relative to these previous observations, the MIPSGAL survey has the advantage of also probing the far infrared emission of the dust. The structure of the nebula as seen in the MIPS $70 \mu \mathrm{m}$ observations is close to that of the shorter wavelengths as seen in the GLIMPSE survey (from 3 to $8 \mu \mathrm{m}$ ): the cloud surface is significantly brighter than the inner shell.

Thanks to the MIPS 24 and MIPS $70 \mu \mathrm{m}$ observations, we have been able to place constraints on the temperature of the grains emitting in the FIR range and the interstellar radiation field intensity required to heat them to these temperatures with our dust model. The dust temperature varies from $\sim 35 \mathrm{~K}$ in the PDRs to $\sim 70 \mathrm{~K}$ in the shell. The required intensity of the radiation field within the PDRs is about an order of magnitude lower than that provided by the star cluster NGC 6611. The shell of hot dust, however, requires an radiation field intensity about a factor of a few higher than that provided by the cluster.

Combining all the IR observations at our disposal into SEDs that sample the whole nebula with our dust model, we have reproduced the observations to constrain both the radiation field intensity and the dust-size distribution. In the PDRs, we have confirmed that the required radiation field intensity is about a few tenths of that provided by NGC 6611. The dust-size distribution is dominated by BGs, even though all the dust components are present with abundances of a factor of a few, at most, different from those of the DHGL medium. In the shell, we have also confirmed that the required radiation field intensity is a factor of a few larger than that of NGC 6611. The PAHs are absent and the VSGs are more abundant, by up to a factor 10, than in the DHGL medium.

Extinction and the dispersion of the stars across the nebula can account for the lower radiation field intensity required for the PDRs. In contrast, an additional source of heating is required for the shell. Neither the spatial variations in the radiation field intensity nor the Lyman alpha photons contribution can account for the discrepancy between the required and provided UV heating of the dust. The precisely determined positions of the stars indicate that Pilbratt's blob is only $0.25 \mathrm{pc}$ from an $08.5 \mathrm{~V}$ star and may thus be a bow shock.

We have thus invoked gas-grain collisions as an extra source of heating. Our modeling leads to a fit to the shell SED that requires a pressure of a few $10^{7} \mathrm{~K} \mathrm{~cm}^{-3}$. This pressure is at least a factor of a few larger than that inferred either from optical observations at the end of the photo-evaporation flow arising from Pillar or from Pilbratt's blob bow shock nature.

We finally discussed two interpretations of the mid-IR shell in the general context of a massive-star forming region. In a first scenario, we proposed that the shell is wind blown by the stars. We found that the star cluster does not provide enough mechanical energy via stellar winds to power the shell emission. Therefore, the shell has been explained in terms of a modified dust grain size distribution (large carbon grains shattered to nanometric sizes) with heating only due to UV emission. The implication is then that massive-star forming regions such as M 16 have a major impact on their dust-size distribution, which can be checked for other similar regions. Alternatively, we have proposed a second scenario, in which the shell is heated by the hidden remnant of a supernova from a very massive progenitor, and for which the dust provides a fast cooling. The implication is then that our observations occur during a short-lived, late stage of evolution of the remnant, which can be checked with new X-ray observations.

The Eagle Nebula IR emission morphology is similar to that of many other star-forming regions observed within the GLIMPSE and MIPSGAL surveys (Churchwell et al. 2006; Carey et al. 2009). For the first time, we have quantitatively discussed this in terms of dust modeling. The work we present would need to be extended to other SFRs with IR morphology similar to that of M 16 to ascertain whether the interpretation would be challenged by the same problem in accounting for the dust temperature. Moreover, future analysis of additional observations (mid-to-far IR spectral mapping from Spitzer/IRS and MIPS-SED, near-IR narrow band imaging from CFHT/WIRCam) of the Eagle Nebula will provide us with more 
constraints on the physical conditions and dust properties in M 16's inner shell.

Acknowledgements. This work is based in part on observations made with the Spitzer Space Telescope, which is operated by the Jet Propulsion Laboratory, California Institute of Technology under a contract with NASA. Support for this work was provided by NASA through an award issued by JPL/Caltech.

\section{References}

Begelman, M. C., \& Fabian, A. C. 1990, MNRAS, 244, 26 Beltrametti, M., Tenorio-Tagle, G., \& Yorke, H. W. 1982, A\&A, 112, 1

Benjamin, R. A., Churchwell, E., Babler, B. L., et al. 2003, PASP, 115, 953

Carey, S. J., Noriega-Crespo, A., Mizuno, D. R., et al. 2009, PASP, 121, 76

Cesarsky, C. J., Abergel, A., Agnese, P., et al. 1996a, A\&A, 315, L32

Cesarsky, D., Lequeux, J., Abergel, A., et al. 1996b, A\&A, 315, L309

Churchwell, E., Povich, M. S., Allen, D., et al. 2006, ApJ, 649, 759

Churchwell, E., Babler, B. L., Meade, M. R., et al. 2009, PASP, 121, 213

Compiègne, M., Abergel, A., Verstraete, L., \& Habart, E. 2008, A\&A, 491, 797

Compiègne, M., Verstraete, L., Jones, A., et al. 2011, A\&A, 525, A103

Désert, F.-X., Boulanger, F., \& Puget, J. L. 1990, A\&A, 237, 215

Draine, B. T. 1981, ApJ, 245, 880

Dufton, P. L., Smartt, S. J., Lee, J. K., et al. 2006a, The VLT-FLAMES Survey of Massive Stars (Dufton+, 2006)

Dufton, P. L., Smartt, S. J., Lee, J. K., et al. 2006b, A\&A, 457, 265

Dwek, E. 1987, ApJ, 322, 812

Dwek, E., Foster, S. M., \& Vancura, O. 1996, ApJ, 457, 244

Everett, J. E., \& Churchwell, E. 2010, ApJ, 713, 592

Fazio, G. G., Hora, J. L., Allen, L. E., et al. 2004, ApJS, 154, 10

Flagey, N., Boulanger, F., Noriega-Crespo, A., Carey, S., \& Mizuno, D. 2009a, in The Evolving ISM in the Milky Way and Nearby Galaxies

Flagey, N., Noriega-Crespo, A., Boulanger, F., et al. 2009b, ApJ, 701, 1450

Galliano, F., Madden, S. C., Jones, A. P., et al. 2003, A\&A, 407, 159

Giard, M., Bernard, J. P., Lacombe, F., Normand, P., \& Rouan, D. 1994, A\&A, 291, 239

Guillard, P., Boulanger, F., Pineau Des Forêts, G., \& Appleton, P. N. 2009, A\&A, 502,515

Guillet, V., Jones, A. P., \& Pineau Des Forêts, G. 2009, A\&A, 497, 145
Hester, J. J., Scowen, P. A., Sankrit, R., et al. 1996, AJ, 111, 2349

Hillenbrand, L. A., Massey, P., Strom, S. E., \& Merrill, K. M. 1993, AJ, 106, 1906

Indebetouw, R., Robitaille, T. P., Whitney, B. A., et al. 2007, ApJ, 666, 321

Jones, A. P. 2004, in Astrophysics of Dust, ed. A. N. Witt, G. C. Clayton, \& B. T. Draine, ASP Conf. Ser., 309, 347

Kraemer, K. E., Shipman, R. F., Price, S. D., et al. 2003, AJ, 126, 1423

Kudritzki, R., \& Puls, J. 2000, ARA\&A, 38, 613

Lefloch, B., Cernicharo, J., Perez-Martinez, S., \& Cesarsky, D. 1999, in The Universe as Seen by ISO, ed. P. Cox, \& M. Kessler, ESA SP, 427, 497

Leitherer, C., Schaerer, D., Goldader, J. D., et al. 1999, ApJS, 123, 3

Linsky, J. L., Gagné, M., Mytyk, A., McCaughrean, M., \& Andersen, M. 2007, ApJ, 654, 347

Markwardt, C. B. 2009, Astronomical Data Analysis Software and Systems XVIII, ASP Conf. Ser., 411, 251

Martayan, C., Floquet, M., Hubert, A. M., et al. 2008, A\&A, 489, 459

Mizuno, D. R., Carey, S. J., Noriega-Crespo, A., et al. 2008, PASP, 120, 1028

Omont, A., Gilmore, G. F., Alard, C., et al. 2003, A\&A, 403, 975

Pilbratt, G. L., Altieri, B., Blommaert, J. A. D. L., et al. 1998, A\&A, 333, L9

Povich, M. S., Stone, J. M., Churchwell, E., et al. 2007, ApJ, 660, 346

Rho, J., \& Borkowski, K. J. 2002, ApJ, 575, 201

Rho, J., Reach, W. T., Lefloch, B., \& Fazio, G. G. 2006, ApJ, 643, 965

Rieke, G. H., Young, E. T., Engelbracht, C. W., et al. 2004, ApJS, 154, 25

Rozyczka, M. 1985, A\&A, 143, 59

Smith, R. K., Krzewina, L. G., Cox, D. P., Edgar, R. J., \& Miller, W. W. I. 1996, ApJ, 473, 864

Squires, G. K., Storrie-Lombardi, L. J., \& Spitzer Legacy Project Team 2005, in BAAS, 37, 1323

Tenorio-Tagle, G., Beltrametti, M., Bodenheimer, P., \& Yorke, H. W. 1982, A\&A, 112, 104

Urquhart, J. S., White, G. J., Pilbratt, G. L., \& Fridlund, C. V. M. 2003, A\&A, 409, 193

van Buren, D., Mac Low, M., Wood, D. O. S., \& Churchwell, E. 1990, ApJ, 353, 570

Vázquez, G. A., \& Leitherer, C. 2005, ApJ, 621, 695

Watson, C., Povich, M. S., Churchwell, E. B., et al. 2008, ApJ, 681, 1341

Watson, C., Corn, T., Churchwell, E. B., et al. 2009, ApJ, 694, 546

Westmoquette, M. S., Slavin, J. D., Smith, L. J., \& Gallagher, III, J. S. 2009, MNRAS, 1793 